\title{
Proteomic phenotyping of stimulated Müller cells uncovers profound pro-inflammatory signaling and antigen-presenting capacity
}

1 Adrian Schmalen ${ }^{1,2}$, Lea Lorenz ${ }^{2}$, Antje Grosche ${ }^{3}$, Diana Pauly ${ }^{4,5}$, Cornelia A. Deeg², Stefanie M. Hauck ${ }^{*}$

${ }^{1}$ Research Unit Protein Science and Metabolomics and Proteomics Core, Helmholtz Center Munich,

4 German Research Center for Environmental Health, D-85764 Neuherberg, Germany

${ }^{2}$ Chair of Physiology, Department of Veterinary Sciences, LMU Munich, D-82152 Martinsried,

6 Germany

$7{ }^{3}$ Department of Physiological Genomics, Biomedical Center, LMU Munich, D-82152 Martinsried,

8 Germany

$9{ }^{4}$ Experimental Ophthalmology, Philipps-University Marburg, D-35043 Marburg, Germany

${ }^{5}$ Department of Ophthalmology, University Hospital Regensburg, D-93053 Regensburg, Germany

11 * Correspondence:

12 Corresponding Author

13 hauck@helmholtz-muenchen.de

14 Keywords: Müller cells, atypical antigen-presenting cell, immune response, diabetic

15 retinopathy, oxidative phosphorylation, complement system, cytokines, retina.

\section{Abstract}

17 Müller cells are the main macroglial cells of the retina exerting a wealth of functions to maintain

18 retinal homoeostasis. Upon pathological changes in the retina, they become gliotic with both protective and detrimental consequences. Accumulating data also provide evidence for a pivotal role of Müller cells in the pathogenesis of diabetic retinopathy (DR). While microglial cells, the resident immune cells of the retina are considered as main players in inflammatory processes associated with DR, the implication of activated Müller cells in chronic retinal inflammation remains to be elucidated. In order to assess the signaling capacity of Müller cells and their role in retinal inflammation, we performed in-depth proteomic analysis of Müller cell proteomes and secretomes after stimulation with INF $\gamma$, TNF $\alpha$, IL-4, IL-6, IL-10, VEGF, TGF $\beta 1$, TGF $\beta 2$ and TGF $\beta 3$. We used both, primary porcine Müller cells and the human Müller cell line MIO-M1 for our hypothesis generating approach. Our results point towards an intense signaling capacity of Müller cells, which reacted in a highly discriminating manner upon treatment with different cytokines. Stimulation of Müller cells resulted in a primarily pro-inflammatory phenotype with secretion of cytokines and components of the complement system. Furthermore, we observed evidence for mitochondrial dysfunction, implying oxidative stress after treatment with the various cytokines. Finally, both MIOM1 cells and primary porcine Müller cells showed several characteristics of atypical antigenpresenting cells, as they are capable of inducing MHC class I and MHC class II with co-stimulatory molecules. In line with this, they express proteins associated with formation and maturation of phagosomes. Thus, our findings underline the importance of Müller cell signaling in the inflamed retina, indicating an active role in chronic retinal inflammation underlying the pathogenesis of diabetic retinopathy. 


\section{$1 \quad$ Introduction}

39 Neurodegenerative diseases of the retina are characterized by progressive photoreceptor damage eventually resulting in vision loss (1). Accumulating evidence over the last years led to the recognition of chronic inflammation as an important part of the pathogenesis underlying this heterogeneous group of retinal diseases (2-4). While the loss of photoreceptors in inherited retinal diseases such as retinitis pigmentosa originates in various genetic mutations (5), the driving force that leads to retinal degeneration and blindness in diabetic retinopathy (DR) is a disturbed metabolism with hyperglycemia and dyslipidemia, resulting in microvascular damage (6). DR is among the most frequent causes of blindness worldwide with a rising prevalence (7). Chronic hyperglycemia in diabetes patients induces the activation of leukocytes in the periphery (8) as well as micro- and macroglial cells in the retina (9-11). This results in the release of pro-inflammatory cytokines and eventually leads to photoreceptor cell death $(12,13)$. Microglial cells, the resident immune cells of the retina, are acknowledged as the main drivers of retinal immune responses (14). However, growing evidence suggests that the interaction of micro- and macroglial cells essentially shapes retinal inflammation and photoreceptor degeneration $(15,16)$.

Retinal Müller glial cells constitute the primary macroglial cells of the retina (17). They span the all retinal neurons (18). While they maintain retinal homeostasis during steady-state conditions, activation of Müller cells under pathological conditions results in gliosis, a cellular attempt to restore insulted tissue, with both protective and detrimental effects (19). It is known that Müller cells are an important source of neurotrophic factors but also of pro-inflammatory and angiogenetic cytokines (20-23). They play an important role in DR pathogenesis $(24,25)$ and it was also suggested that they are involved in retinal immune responses (26-30). However, the impact of their protective or detrimental effects on retinal inflammation in DR and other neurodegenerative retinal diseases remains elusive so far. Thus, we performed an in-depth-analysis of the Müller cell proteome and secretome after stimulation with various pro- and anti-inflammatory cytokines as well as growth factors. For our analysis, we used cells of the human Müller glia cell-line MIO-M1 as well as primary porcine retinal Müller Glia (pRMG), as porcine eyes resemble human eyes regarding their anatomy (31). Furthermore, the pig is established as a useful model for research in diabetic retinopathy $(32,33)$. Comparative quantitative proteomic analysis allows to elucidate key proteins or pathways involved in disease pathogenesis. In addition, this approach enables the discovery of new biomarkers and has therefore proven as a valuable tool for deciphering pathophysiological key mechanisms $(34,35)$. Our hypothesis-generating study yielded comprehensive data on the capacity of Müller cells to react in a very differentiated manner to varying stimulants. In addition to the secretion of pro-inflammatory cytokines, we observed expression of MHC class I and II molecules and proteins that are associated with the processing of antigens. We therefore propose that Müller cells are critical modulators of the retinal immune response and might exert an antigen-presenting function. Thus, attention should be paid to their implication in chronic inflammation underlying degenerative retinal diseases.

\section{Materials and methods}

\subsection{Cell preparation and culture}

79

Cells were maintained in Dulbecco's modified eagle medium (DMEM) containing 10\% ( $v / v)$ 
81

82

83

84

85

86

87

88

89

90

91

92

93

94

95

96

97

98

99

100

101

102

103

104

105

106

107

108

109

110

111

112

113

114

115

116

117

118

119

120

121

122

123

124

otherwise stated. Cell culture media and reagents were purchased from Gibco (Life Technologies $\mathrm{GmbH}$, Darmstadt, Germany).

Ten porcine eyes from healthy pigs were kindly provided from a local abattoir. The use of porcine material from the abattoir was approved for purposes of scientific research by the appropriate board of the veterinary inspection office, Munich, Germany (registration number DE 09162 0008-21). No experimental animals were involved in this study. Within $2 \mathrm{~h}$ after enucleation, eyes were processed under a laminar flow hood under sterile conditions as previously described $(30,36)$. In short, periocular tissue was removed and the eyeballs were rinsed in $80 \%$ ethanol followed by washing with cold PBS. Afterwards, eyeballs were stored in DMEM until further processing. The eyeballs were opened circumferentially parallel to the limbus corneae, and anterior parts of the eyes were discarded. The retina was detached from the posterior eyeballs and transferred into a petri dish containing DMEM. After removal of vitreous and pigment epithelium residues, major blood-vessels were excised and the remaining retinal tissue was cut into very small fragments using micro-scissors. Resulting fragments were washed in Ringer's solution followed by enzymatic digestion at $37^{\circ} \mathrm{C}$ with papain previously activated by incubation with $1.1 \mu \mathrm{M}$ EDTA, $0.067 \mu \mathrm{M}$ mercaptoethanol and 5.5 $\mu \mathrm{M}$ cysteine-HCl. Enzymatic digestion was stopped after $12 \mathrm{~min}$ by adding serum-containing DMEM, followed by addition of Desoxyribonuclease I (Sigma-Aldrich Chemie GmbH, Taufkirchen, Germany) and trituration. After sedimentation of the cells, the supernatant was carefully removed using Pasteur pipettes. The remaining pellets were resuspended in DMEM, pooled and seeded into 6well plates (Sarstedt, Nümbrecht, Germany). The following day, thorough panning of the plates and removal of the supernatant were performed in order to eliminate non-attached cells, yielding pure Müller cell cultures as previously described $(37,38)$. Cells were cultured at $37{ }^{\circ} \mathrm{C}$ and $5 \% \mathrm{CO}_{2}$ with regular exchange of medium and repeated microscopic control of cell density and purity according to previous reports $(37,39)$.

The human Müller cell line Moorfields/Institute of Ophthalmology-Müller 1 (MIO-M1; RRID:CVCL_0433) was a kind gift of G. A. Limb (39). They were tested negative for mycoplasma contamination. Two days before treatment, $1 \times 10^{5} \mathrm{MIO}-\mathrm{M} 1$ cells per well were seeded in 6 -well plates and incubated at $37^{\circ} \mathrm{C}$ and $5 \% \mathrm{CO}_{2}$ until further processing.

\subsection{Cell stimulation}

The human cytokines Interleukin 10 (IL-10), Transforming Growth Factor beta-1 (TGF $\beta 1$ ), Transforming Growth Factor beta-2 (TGF $\beta 2$ ), Transforming Growth Factor beta-3 (TGF $\beta 3$ ), and Tumor Necrosis Factor Alpha (TNF $\alpha$ ) were purchased from Sigma-Aldrich, Interleukin 4 (IL-4) and Interferon Gamma (IFNy) from R\&D Systems/Bio-Techne (R\&D Systems/Bio-Techne, WiesbadenNordenstadt, Germany), and Interleukin 6 (IL-6) and Vascular Endothelial Growth Factor 165 (VEGF) from PeproTech (PeproTech, Winterhude, Germany). Porcine TGF $\beta 3$ was purchased from Biozol (Biozol, Eching, Germany), whereas porcine TGF $\beta 1$, TGF $\beta 2$, IL-4, IL-6, IL-10, IFNy and TNF $\alpha$ were from R\&D System/Bio-Techne. Since there was no porcine VEGF available, the above mentioned human VEGF was also used for stimulation of pRMG.

To diminish the influence of cytokines present in FCS, both confluent pRMG and MIO-M1 cells were rinsed two times with prewarmed serum-free medium, followed by starvation for $1 \mathrm{~h}$ at $37^{\circ} \mathrm{C}$ and 5\% $\mathrm{CO}_{2}$ with serum-deprived medium. Afterwards, cells were treated over night with IFNy, IL4, IL-6, IL-10, TGF $\beta 1$, TGF $\beta 2$, TGF $\beta 3$, TNF $\alpha$ or VEGF 165 , respectively, in a randomized plate design at a concentration of $5 \mathrm{ng} / \mathrm{mL}$ in medium without FCS. Untreated cells cultured in serum-free medium served as a control. 


\subsection{Sample collection and proteolysis}

126 Supernatants were collected $24 \mathrm{~h}$ after treatment, passed through medium equilibrated $0.2 \mu \mathrm{m}$

127 Millex-GP filter units (Merck Chemicals GmbH, Darmstadt, Germany), and transferred into $2 \mathrm{~mL}$

128 Lo-Bind tubes (Eppendorf AG, Hamburg, Germany). Afterwards, cells were washed once with

129 DPBS. $200 \mu$ L RIPA buffer containing Roche cOmplete Mini Protease Inhibitor Cocktail (Merck

130 Chemicals $\mathrm{GmbH}$ ) was applied directly into each well and cells were detached with a cell scraper.

131 Lysates were transferred into freshly prepared 1.5 mL Lo-Bind tubes (Eppendorf AG). Protein

132 concentration of the lysates was determined by Pierce BCA assay (Thermo Fisher Scientific). Ten $\mu \mathrm{g}$

133 protein per lysate or $400 \mu \mathrm{L}$ supernatant per sample were digested with Lys-C and trypsin using a

134 modified FASP procedure as described elsewhere (40, 41).

\section{$135 \quad 2.4 \quad$ LC-MS/MS and quantitative analysis}

136 LC-MSMS analysis was performed on a QExactive HF mass spectrometer (Thermo Fisher Scientific) 137 online coupled to a UItimate 3000 RSLC nano-HPLC (Dionex, Sunnyvale, USA). Samples were

138 automatically injected and loaded onto a C18 trap column for $5 \mathrm{~min}$. Afterwards, samples were eluted and separated on a C18 analytical column (Acquity UPLC M-Class HSS T3 Column, $1.8 \mu \mathrm{m}, 75 \mu \mathrm{m}$ $\times 250 \mathrm{~mm}$; Waters, Milford, USA). Samples were separated by a $95 \mathrm{~min}$ non-linear acetonitrile gradient at a flow rate of $250 \mathrm{nl} / \mathrm{min}$. Resolution of the MS spectra was recorded at 60,000 with an AGC target of $3 \times 10^{6}$ and a maximum injection time of $50 \mathrm{~ms}$ from 300 to $1,500 \mathrm{~m} / \mathrm{z}$. The 10 most abundant peptide ions were selected from the MS scan and fragmented via HCD. Thereby, the normalized collision energy was 27 with an isolation window of $1.6 \mathrm{~m} / \mathrm{z}$, and a dynamic exclusion of $30 \mathrm{~s}$. MS/MS spectra were recorded at a resolution of 15,000 with an AGC target of $10^{5}$ and a maximum injection time of $50 \mathrm{~ms}$. Spectra with unassigned charges, and charges of +1 and $>8$ were excluded from the precursor selection.

The four datasets (lysates of pRMG and MIO-M1 cells, and secretomes of pRMG and MIO-M1 cells) were analyzed separately. The Proteome Discoverer 2.4 SP1 software (version 2.4.1.15; Thermo Fisher Scientific) was used for peptide and protein identification via a database search (Sequest HT search engine) against the SwissProt Human (MIO-M1) and Ensembl Pig (pRMG). Database search was performed with full tryptic specificity, allowing for up to one missed tryptic cleavage site, a precursor mass tolerance of $10 \mathrm{ppm}$, and fragment mass tolerance of $0.02 \mathrm{Da}$. Carbamidomethylation of Cys was set as a static modification. Dynamic modifications included deamidation of Asn and Gln, oxidation of Met, and a combination of Met loss with acetylation on protein N-terminus. Peptide spectrum matches and peptides were validated with the Percolator algorithm (42). Each dataset was measured as triplicate. One control sample in the dataset of MIOM1 secretomes had an unexpected low overall abundance and was excluded as an outlier. Only the top-scoring hits for each spectrum were accepted with a false discovery rate (FDR) $<1 \%$ (high confidence). The final list of proteins satisfying the strict parsimony principle included only protein groups passing an additional protein confidence filter FDR $<5 \%$ filter (target/decoy concatenated search validation).

163 Quantification of proteins, after precursor recalibration, was based on intensity values (at RT apex) 164 for all unique peptides per protein. Peptide abundances were normalized to the total peptide amount. The protein abundances were calculated summing the normalised abundance values of corresponding unique peptides. These protein abundances were used for calculation of enrichment ratios of proteins in the stimulated samples to the untreated samples, resulting in single ratios for every quantified protein in every treated sample. Significance of the ratios was tested using a background-based t-test with correction for multiple testing according to Benjamini-Hochberg (adjusted $P$-value) $(43,44)$. 


\subsection{Data analysis and visualization}

171 Calculation of the abundance ratio weight requires abundance values for both, the stimulated sample

172 and the control. However, if a protein is exclusively expressed in one of these samples, the Proteome

173 Discoverer software fails to calculate a respective abundance ratio weight. Since these extreme

174 values were of special interest to us, the missing abundance ratio weights were imputed using the $\mathrm{R}$

175 package mice (version 3.13.0) and the "classification and regression trees" imputation method.

176 Ingenuity Pathway Analysis (IPA; Qiagen, Hilden, Germany) was used to analyze overrepresentation

177 of proteins in canonical pathways of the IPA library, as described elsewhere (45). IPA allows

178 deducing potential physiological effects of the various tested cytokines. Analysis was performed

179 based on the fold-change of the stimulated samples and the abundance ratio $P$-value. Fisher's exact

180 test allowed testing for nonrandom associations of proteins in the datasets and the different canonical

181 pathways (46). Furthermore, the method of Benjamini-Hochberg (B-H $P$-value) corrected for

182 multiple testing (44).

183 The euclidean distance for the heatmap analysis was calculated with the open source software Cluster

1843.0 and hierarchically clustered by complete linkage clustering (47). The resulting heat map was

185 visualized with the open source software Java Treeview (version 1.2.0) (48).

\section{Results}

\section{3.1 Differential secretion of proteins after stimulation of Müller cells with various cytokines}

188 Müller cells are in close contact to all retinal cells, the vitreous and the blood vessels (17). To address, whether this privileged position within the retina also translates into extensive signaling between Müller cells and the surrounding cells, the secretomes of the human Müller cell-derived cell line MIO-M1 and of pRMG were quantitatively analyzed by mass spectrometry after stimulation for $24 \mathrm{~h}$ with the cytokines IFN $\gamma$, IL-10, IL-4, IL-6, TGF $\beta 1$, TGF $\beta 2$, TGF $\beta 3$, TNF $\alpha$ and VEGF, respectively. By this means, we quantified 2,031 proteins in the supernatant of MIO-M1 cells (Suppl. Table 1) and 3,093 proteins in the supernatant of pRMG across all treatment groups (Suppl. Table 2). 
A MIO-M1 Secretome: IFNY

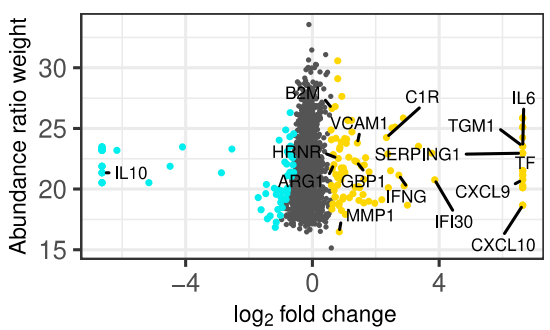

D MIO-M1 Secretome: IL-4

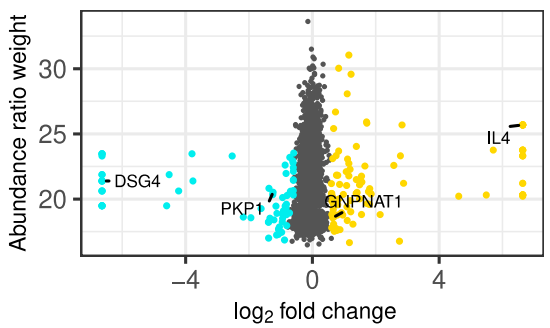

G MIO-M1 Secretome: TGF $\beta 1$

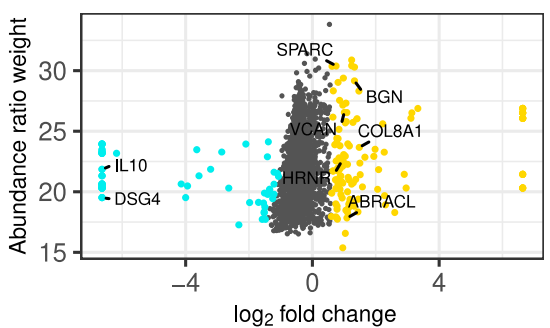

B MIO-M1 Secretome: TNF $\alpha$

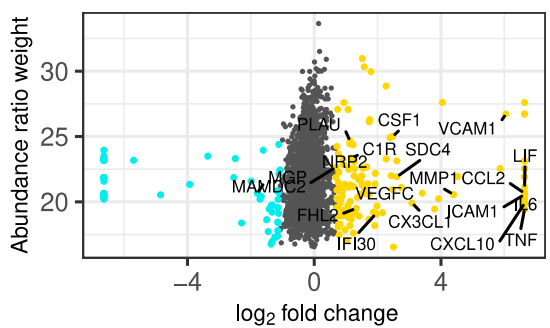

E MIO-M1 Secretome: IL-6

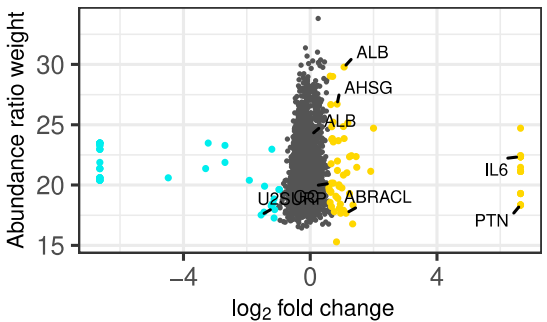

H MIO-M1 Secretome: TGF $\beta 2$

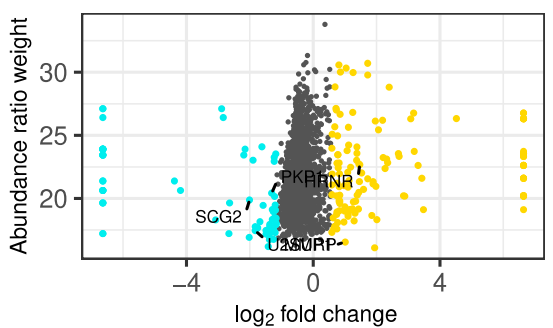

C MIO-M1 Secretome: VEGF

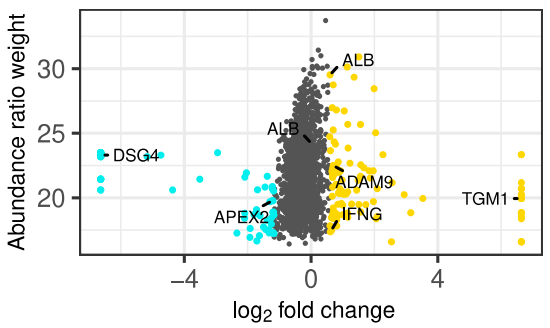

F MIO-M1 Secretome: IL-10

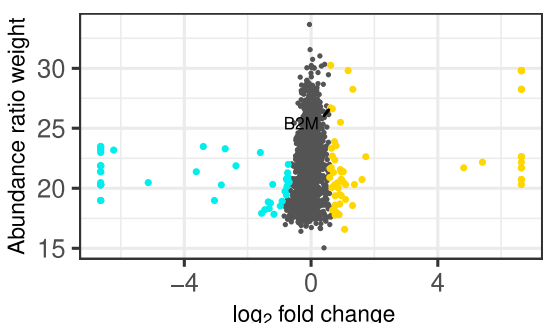

I MIO-M1 Secretome: TGF 33

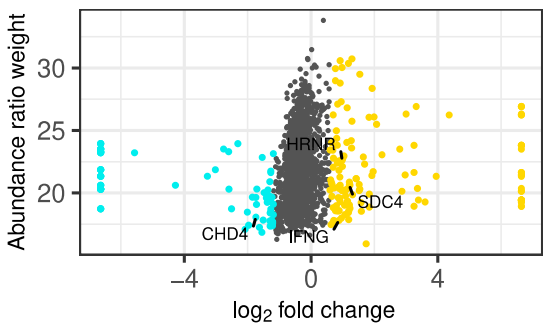

Figure 1. Scatterplot of all identified proteins from MIO-M1 secretomes after treatment with the indicated cytokines for $24 \mathrm{~h}(\mathbf{A}-\mathbf{I})$. Proteins with significant changes in their abundance $\left( \pm \log _{2}(1.5)\right.$ fold expression, corrected $p$-value $\left.\leq 0.05\right)$ were colored, with upregulated proteins being depicted as yellow dots, while down-regulated proteins are colored cyan. Proteins with significantly altered abundance in both, MIO-M1 and pRMG secretomes, are labeled with their gene symbol. Keratins were excluded.

Fig. 1 and Suppl. Fig. S1 summarize changes in the secretome after treatment of Müller cells with the different cytokines. A $\log _{2}$ fold change of \pm 0.58 and a corrected $p$-value of equal or less than 0.05 served as cutoff to define significantly upregulated or downregulated genes, respectively. Proteins equally regulated in MIO-M1 cells and pRMG were labeled with their gene symbol. However, this was only possible for proteins with identical gene symbols in the human and the porcine protein database. After treatment with IFN $\gamma, 107$ proteins in the secretome of MIO-M1 cells and 176 proteins in the secretome of pRMG were significantly more abundant, while 67 proteins of MIO-M1 cells and 96 proteins of pRMG were significantly less abundant in the supernatants (Fig. 1A; Suppl. Fig. S1A). Intriguingly, MIO-M1 cells and pRMG shared 21 upregulated and one downregulated protein. Among these shared regulated proteins after treatment with IFN $\gamma$ were many with immune system functions, like signaling molecules (e.g. C-X-C Motif Chemokine Ligand 9 (CXCL9), CXCL10, IL6) and components of the complement system (e.g. C1R, Serpin Family G Member 1 (SERPING1)). Upon treatment with TNFa, 127 (MIO-M1) or 143 (pRMG) proteins were more abundant and 57 (MIO-M1) or 87 (pRMG) proteins were less abundant in the supernatant (Fig. 1B, Suppl. Fig. S1B). Within these groups, MIO-M1 cells and pRMG shared 20 upregulated and three downregulated proteins, again with many pro-inflammatory proteins like $\mathrm{C}-\mathrm{X} 3-\mathrm{C}$ Motif Chemokine Ligand 1

This is a provisional file, not the final typeset article 
218 (CX3CL1), CXCL10, C-C Motif Chemokine Ligand 2 (CCL2), IL-6, and C1R being upregulated.

219 Thus, IFN $\gamma$ and TNF $\alpha$ resulted in the most conserved changes of the secretome of Müller cells when comparing between stimulated MIO-M1 cells and pRMG. In contrast, MIO-M1 cells and pRMG only shared ten proteins that were more abundant and three which were less abundant after treatment with VEGF, with seven proteins being keratins (Fig. 1C, Suppl. Fig. S1C). Treatment of Müller cells with the three different interleukins had a subtle influence on secreted proteins with conserved regulation of seven (IL-4), nine (IL-6), and two (IL-10) proteins in the secretome of MIO-M1 cells and pRMG (Fig. 1D-F, Suppl. Fig. S1D-F).

Finally, TGF $\beta$ s led to pronounced alterations in the secretome of MIO-M1 cells. TGF $\beta 1$ enhanced the secretion of 125 proteins while simultaneously reducing the abundance of 67 proteins, TGF $\beta 2$ increased the abundance of 131 proteins while impairing secretion of 69 proteins, and TGF $\beta 3$ raised abundance of 135 proteins and reduced the abundance of 76 proteins (Fig. 1G-I). Furthermore, eleven proteins of MIO-M1 cells and pRMG were similarly regulated by TGF $\beta 1$, eight upregulated and three downregulated (Suppl. Fig. S1G). After treatment with TGF 32 , three proteins were more abundant and four proteins less abundant in the secretome of both cell types (Suppl. Fig. S1H). Additionally, the secretome of TGF $\beta 3$ treated MIO-M1 cells and pRMG shared one downregulated protein and eight upregulated proteins, with most proteins like Osteonectin (Secreted Protein Acidic And Rich In Cysteine; SPARC), Matrix Metallopeptidase 1 (MMP1), Biglycan (BGN) and various keratins being functionally related to extracellular matrix organization (Suppl. Fig. S1I). Furthermore, stimulation with TGF $\beta 3$ evoked upregulation of pro-inflammatory cytokine IFN $\gamma$ in both, the MIO-M1 cell line and pRMG. Overall, there are subtle, but intriguing differences in protein abundances after stimulation of Müller cells with the different TGF $\beta$ isoforms. Some of the proteins differentially induced by the TGF $\beta$ isoforms in MIO-M1 cells are pro-inflammatory cytokines, like IFN $\gamma$, TNF $\alpha$, and CCL2 (Suppl. Table 1). 


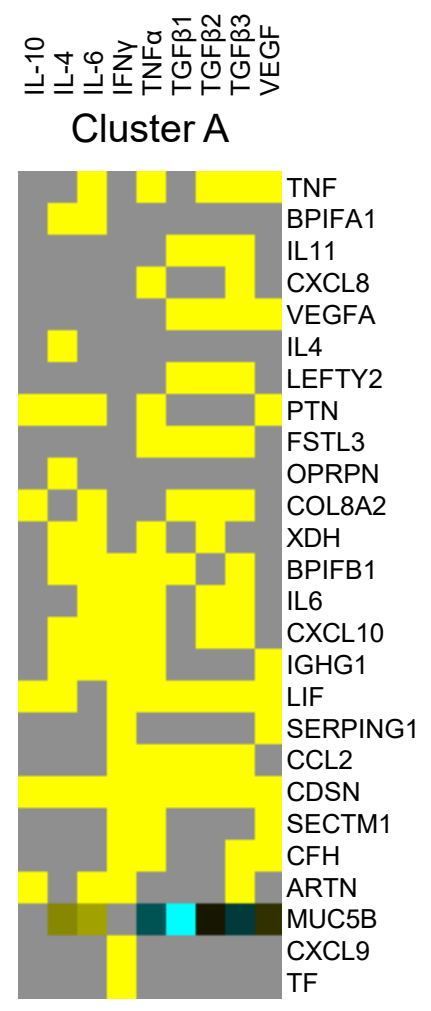

Cluster B

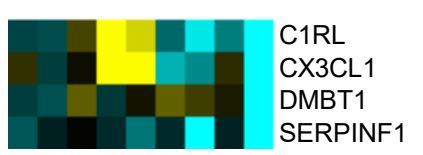

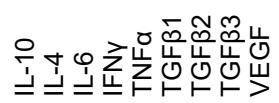

Cluster $C^{\prime}$

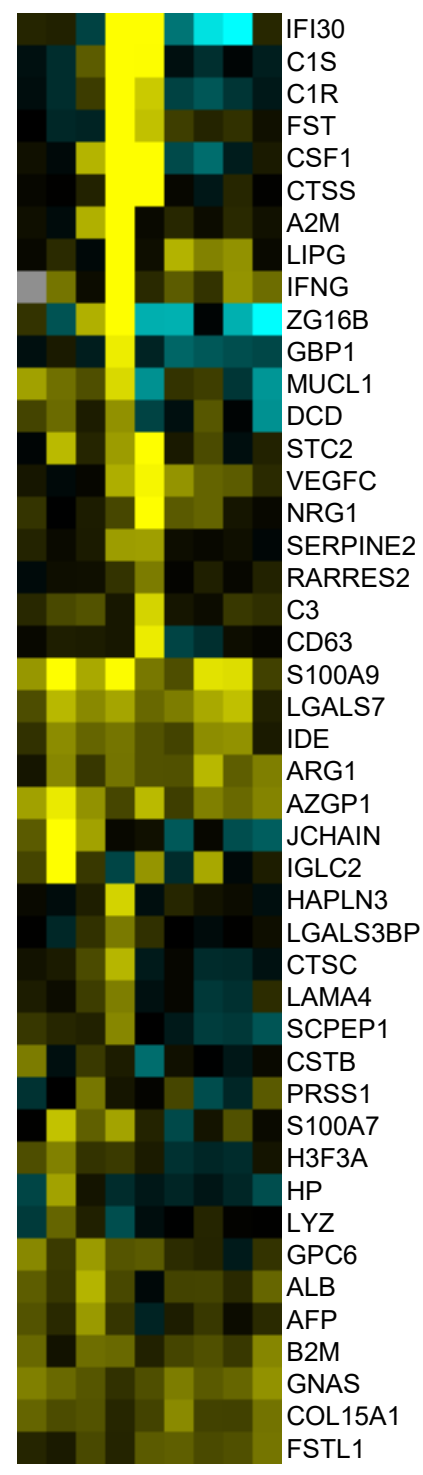

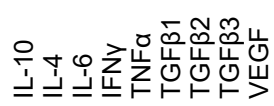

Cluster C"

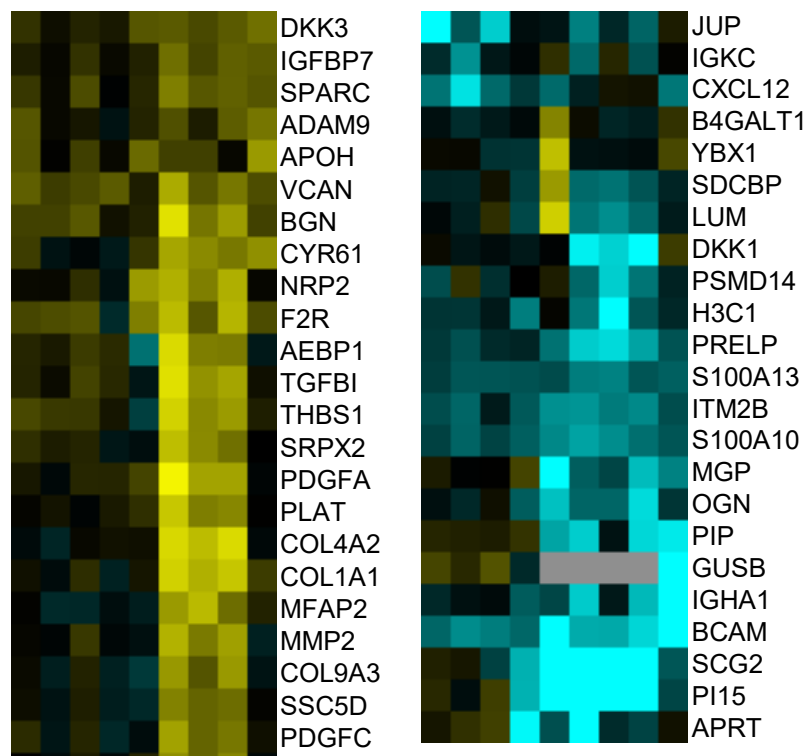

Cluster $\mathrm{E}$

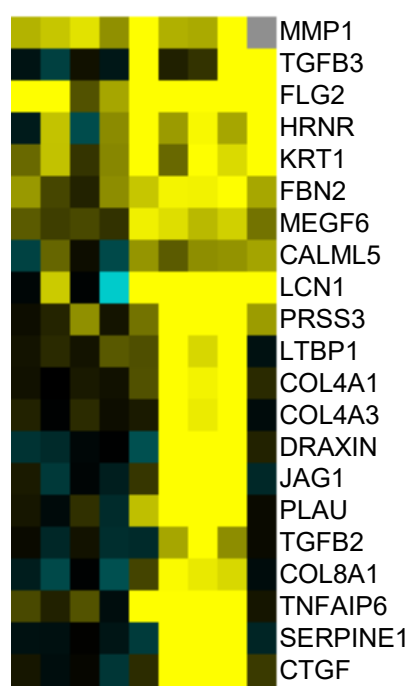

Cluster F

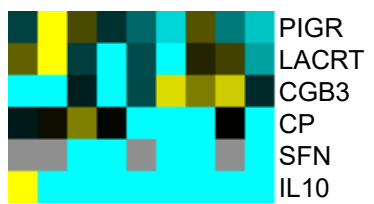

Figure 2. Heatmap of hierarchical cluster analysis of proteins secreted by MIO-M1 cells after treatment with various cytokines. Identified proteins were filtered for extracellular proteins with significant changes in expression $\left( \pm \log _{2}(1.5)\right.$ fold expression, corrected $p$-value $\left.\leq 0.05\right)$. Down-regulated proteins are presented in cyan, while up-regulated proteins are depicted yellow 
untreated control, nor in the respective treatment. Clusters were defined using the branches of a dendrogram and shown as close up with the corresponding gene symbols.

251 Therefore, we ordered secreted proteins in distinct clusters, grouped by similar expression patterns.

252 From the identified proteins of the MIO-M1 supernatant, all cytosolic contaminants were removed,

253 resulting in a list that exclusively contained extracellular proteins. Furthermore, only those proteins

254 that showed a significant change of abundance (corrected $p$-value $\leq 0.05$ and \pm 1.5 -fold abundance) in at least one treatment were selected for further analysis. As a result, a hierarchical heat map and an associated dendrogram consisting of 171 proteins was generated using the $\log _{2}$ abundance ratio of treated and untreated control cells (Suppl. Fig. S2). Furthermore, clusters were highlighted depending on their position on the dendrogram (Fig. 2). To avoid single protein clusters, the proteins Polymeric Immunoglobulin Receptor (PIGR) and Lacritin (LACRT) were assigned to Cluster F, despite being on different branches of the dendrogram.

261 Notably, we identified proteins which were secreted by MIO-M1 cells exclusively after stimulation 262 (cluster A), while another set of proteins was completely absent in MIO-M1 cells (cluster B) treated with VEGF. The largest cluster comprises proteins upregulated by at least one cytokine (cluster C). In contrast to cluster D, consisting of proteins downregulated by TGF $\beta$ s, TGF $\beta$ s induce secretion of proteins of cluster E. Finally, secretion of most proteins of cluster F is deterred by at least one

266 cytokine. 
A

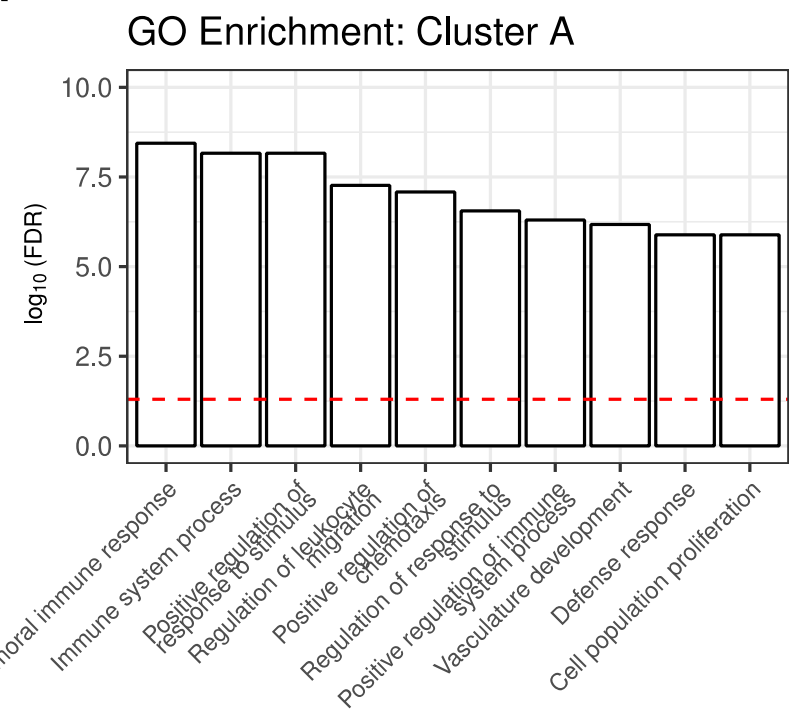

B

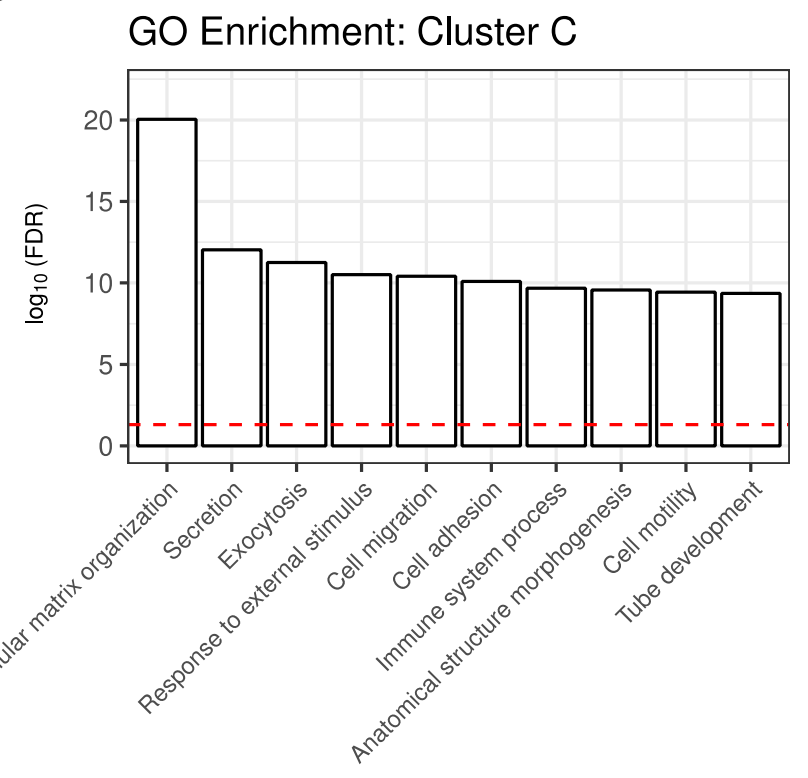

C

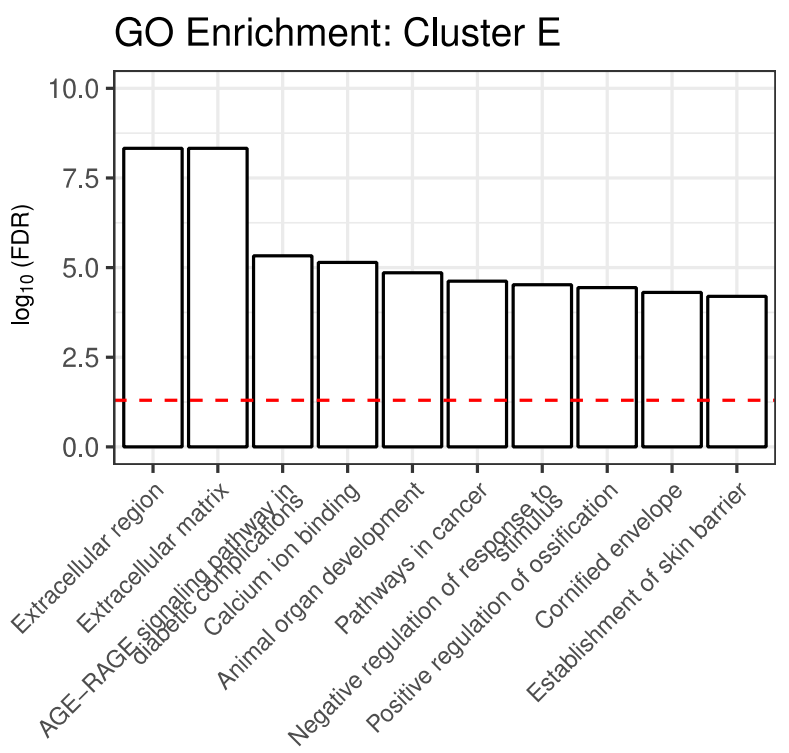

Figure 3. A shiny GO enrichment analysis for the previously defined cluster A (A), cluster C (B) and cluster E (C) was performed. Redundant pathways were reduced to show only one representative pathway. Depicted is the $-\log 10$ (FDR) of the top 10 pathways for each cluster. The red dashed line indicates the significance threshold.

The clusters A, C and E are large clusters with proteins upregulated by at least one cytokine, with cluster A being the cluster with the most pronounced changes in secretion of its members and cluster $\mathrm{C}$ being the most diffuse one. In order to deduce the physiological functions of the secreted proteins of each of these clusters, we performed gene ontology (GO) analysis. Thereby, redundant pathways were condensed to a single representative pathway and the top 10 pathways with the lowest enrichment FDR were displayed. Intriguingly, the top 10 pathways for cluster A were related to 
immune system processes, with "Humoral immune response" and "Immune system process" being the most significant pathways with an enrichment FDR of $3.6 \times 10^{-9}$ and $6.9 \times 10^{-9}$, respectively (Fig. $3 \mathrm{~A})$. Furthermore, cluster $\mathrm{C}$ contained the pathway "Immune system process", while most other pathways in this cluster $\mathrm{C}$ were involved in shaping the extracellular environment (Fig. 3B). Besides further pathways involved in extracellular remodeling, the proteins of cluster E were also associated to the pathway "AGE-RAGE signaling pathway in diabetic complications" (Fig. 3C).

\subsection{In-depth analysis of the Müller cell proteome after stimulation with a selection of cytokines}

Our secretome analysis hints towards extensive signaling between Müller cells and their cellular environment, differentially induced by various cytokines. To elucidate the underlying cellular alterations, we also investigated differences in the proteome of MIO-M1 cells and pRMG cells by mass spectrometry after treatment with these cytokines for $24 \mathrm{~h}$. In total, 5,514 proteins were quantified in the lysates of MIO-M1 cells (Suppl. Table 3) and 4,187 proteins in the lysates of pRMG cells (Suppl. Table 4) across all treatment groups.
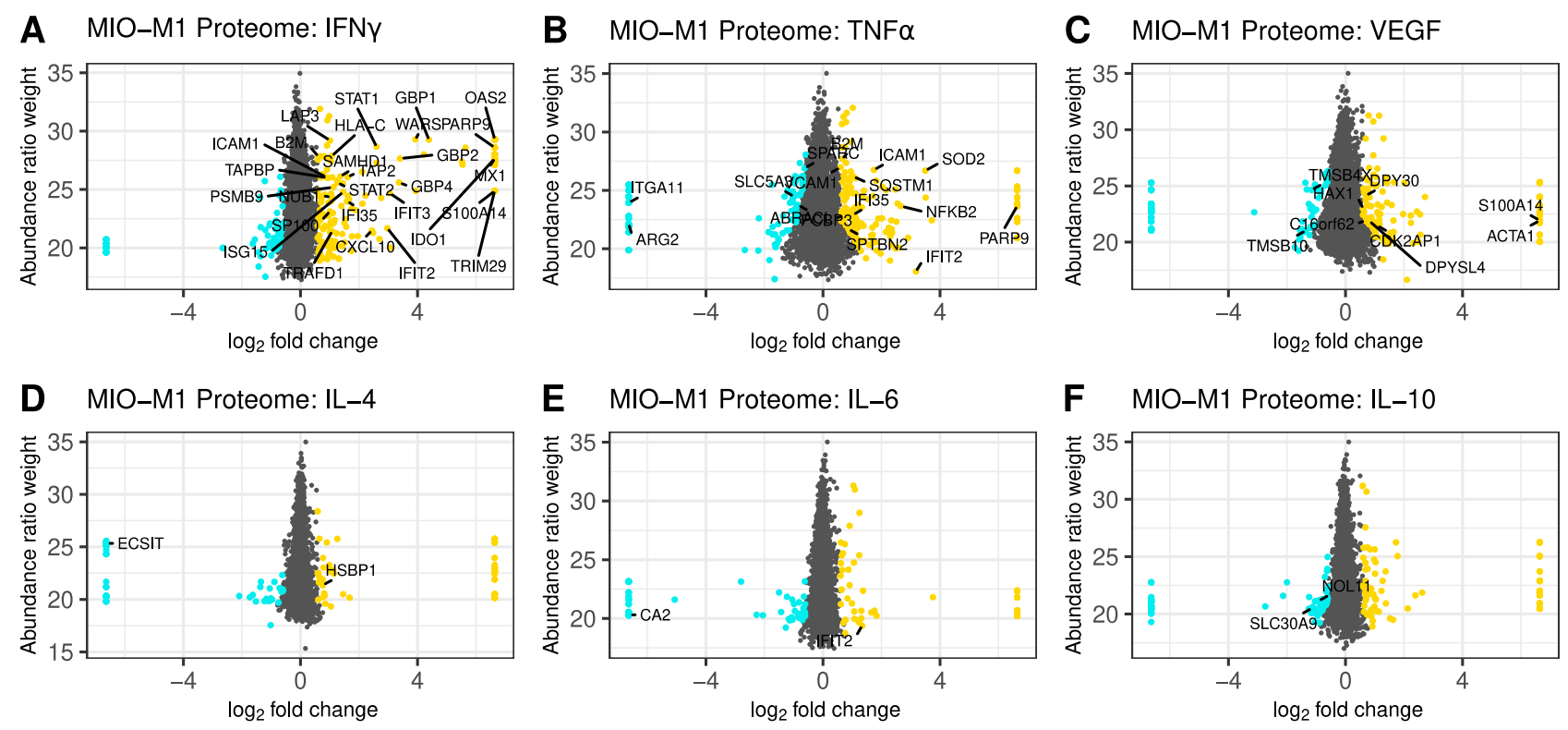

G MIO-M1 Proteome: TGF $\beta 1$
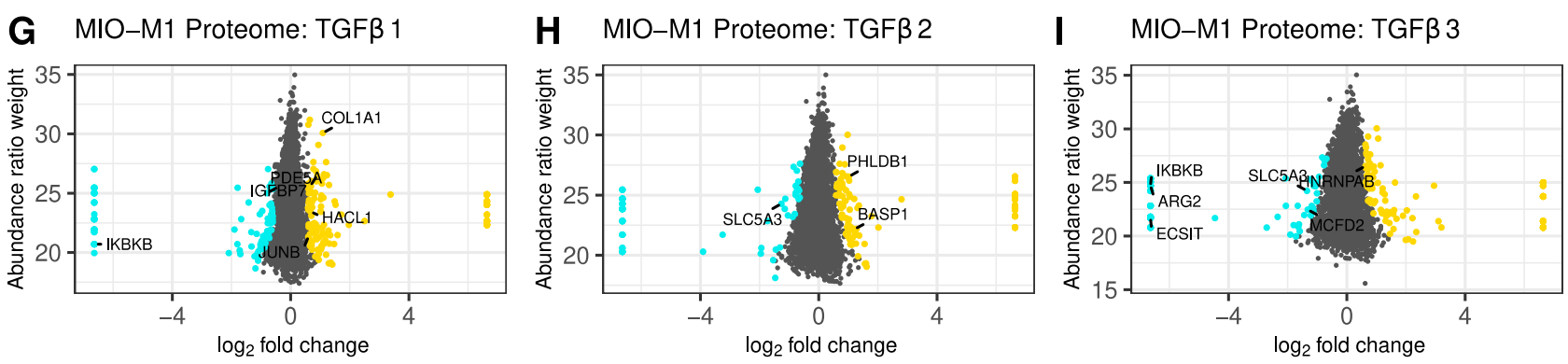

Figure 4. Scatterplot of all identified proteins from MIO-M1 lysates after treatment with the indicated cytokines for $24 \mathrm{~h}$ (A-I). Proteins with significant changes in their abundance $\left( \pm \log _{2}(1.5)\right.$ fold expression, corrected $p$-value $\left.\leq 0.05\right)$ were colored, with upregulated proteins being depicted as yellow dots, while down-regulated proteins are colored cyan. Proteins with 

gene symbol. Keratins were excluded.

The threshold for significant abundance changes was set using the same cutoff values as for the secretome, and equally regulated proteins in MIO-M1 cells and pRMG were labeled with their gene symbol, if they shared the same gene symbol in the human and the porcine database (Fig. 4; Suppl Fig. S3). Although the porcine protein database contains mostly humanized gene symbols, the swine leukocyte antigens (SLA) genes show little sequence homology between the human and the porcine genome and cannot properly be humanized (49). However, Human Leukocyte Antigen-C Alpha Chain (HLA-C) was part of the porcine protein database.

Treatment of Müller cells with IFN $\gamma$ resulted in 206 more abundant and 88 less abundant proteins in MIO-M1 cells and 331 more abundant and 36 less abundant proteins in pRMG lysates (Fig. 4A; Suppl Fig. S3A). Thereof, 29 proteins showed higher expression levels in both cells types. Among the overlapping proteins were restriction factors like SAM And HD Domain Containing Deoxynucleoside Triphosphate Triphosphohydrolase 1 (SAMHD1) and MX Dynamin Like GTPase 1 (MX1), transcription factors like Signal Transducer And Activator Of Transcription 1 (STAT1) and STAT2, regulators of protein homeostasis like Leucine Aminopeptidase 3 (LAP3) or the Proteasome 20S Subunit Beta 9 (PSMB9), and proteins associated with peptide transport and antigen presentation like Transporter 2, ATP Binding Cassette Subfamily B Member (TAP2), TAP Binding Protein (TAPBP), Beta-2-Microglobulin (B2M), as well as HLA-C. After treatment with TNFa, 204 proteins were more abundant in the proteome of MIO-M1 cells, while 119 proteins were less abundant (Fig. 4B). In pRMG, 207 proteins with higher abundance and 285 proteins with lower abundance were identified upon treatment with TNFa, with 18 proteins that were differentially regulated in both cell types (Suppl. Fig. S3B). Among shared proteins that were more abundant after treatment with TNF $\alpha$ were pro-inflammatory proteins like B2M and Nuclear Factor Kappa B Subunit 2 (NFKB2), or adhesion molecules like Intercellular Adhesion Molecule 1 (ICAM1) or Vascular Cell Adhesion Molecule 1 (VCAM1). VEGF led to 143 more and 102 less abundant proteins in MIO-M1 cells or 232 more and 224 less abundant proteins in pRMG, respectively (Fig. 4C; Suppl. Fig. S3C). Thereof, MIO-M1 cells and pRMG shared nine more abundant proteins, inter alia proteins associated with reorganization of the cortical cytoskeleton like Alpha-Actin-1 (ACTA1) or HCLS1 Associated Protein X-1 (HAX1), and two less abundant proteins, Thymosin Beta 10 (TMSB10) and Thymosin Beta 4 X-Linked (TMSB4X), both inhibitors of actin polymerization. Upon treatment with interleukins IL-4, IL-6 and IL-10, the Müller cell proteomes mirrored the subtle effects of these cytokines on the abundance of proteins observed for the Müller cell secretomes (Fig. 4D-F, Suppl. Fig. S3D-F). Also in line with the secretome data, the overlap between differentially abundant proteins of the MIO-M1 and pRMG proteome after treatment with the various interleukins contained only few proteins. In contrast, TGF $\beta 1$ increased the abundance of 143 proteins, while decreasing the abundance of 94 proteins in the proteome of MIO-M1 cells and increased the abundance of 203 proteins, while decreasing the abundance of 103 proteins in the proteome of pRMG (Fig. 4G; Suppl. Fig. S3G). In comparison to the lower abundant proteins Phosphodiesterase 5A (PDE5A) and Inhibitor Of Nuclear Factor Kappa B Kinase Subunit Beta (IKBKB), the proteins Collagen Type I Alpha 1 Chain (COL1A1), Insulin Like Growth Factor Binding Protein 7 (IGFBP7), JunB ProtoOncogene (JUNB), and 2-Hydroxyacyl-CoA Lyase 1 (HACL1) were more abundant in both, MIOM1 cells and pRMG after treatment with TGF $\beta 1$. Following treatment with TGF $\beta 2,125$ proteins of the proteome of MIO-M1 cells and 266 proteins of the proteome of pRMG were more abundant, whereas 67 proteins of the MIO-M1 proteome and 229 proteins of the pRMG proteome were less abundantly expressed (Fig. 4H; Suppl. Fig. S3H). In the case of treatment with TGF 33 , 130 proteins in the MIO-M1 proteome and 185 in the pRMG proteome showed higher abundances, while 94 
344 proteins in MIO-M1 proteome and 250 in the pRMG proteome were less abundant (Fig. 4I; Suppl. 345 Fig. S3I). The overlap of MIO-M1 cells and pRMG treated with TGF $\beta 2$ comprised three proteins, 346 and treatment with TGF $\beta 3$ resulted in an overlap of seven proteins. Overall, pRMG reacted more 347 pronounced to treatment with the various cytokines compared to MIO-M1 cells.

\section{$348 \quad 3.3$ Canonical pathways enriched in Müller cells upon stimulation}

349 Treatment with various cytokines partly induced pronounced changes in the secretome and proteome 350 of Müller cells. In the secretome, these changes primarily included the secretion of pro-inflammatory 351 cytokines and proteins associated with organization of the extracellular matrix. To elucidate overrepresented mechanisms and pathways in stimulated Müller cells, we performed Ingenuity pathway analysis (IPA). We limited the IPA to significantly regulated proteins ( $p$-value $\leq 0.05$ )

354 identified in the MIO-M1 and pRMG lysates. Since IPA cannot handle porcine gene symbols, we 355 replaced the only canonical SLA gene SLA-1 in our pRMG dataset by the canonical human HLA gene HLA-A. Hence, an IPA core analysis was performed with 1,543 proteins for pRMG and with 2,262 proteins for the MIO-M1 cells. IPA identified 338 canonical pathways within the proteome of MIO-M1 cells and 218 canonical pathways in the proteome of pRMG that were significantly 359 enriched by at least one of the used cytokines (IPA p-value $\leq 0.05$; Suppl. Table 5). Among the 360 identified canonical pathways were many pathways associated with signaling, cell death, immune system processes and the cellular redox state (Fig. 5; Suppl. Fig. S4). A selection of canonical pathways enriched in both pRMG and MIO-M1 cells after treatment with at least one of the tested

363 cytokines is depicted in Fig. 5. 


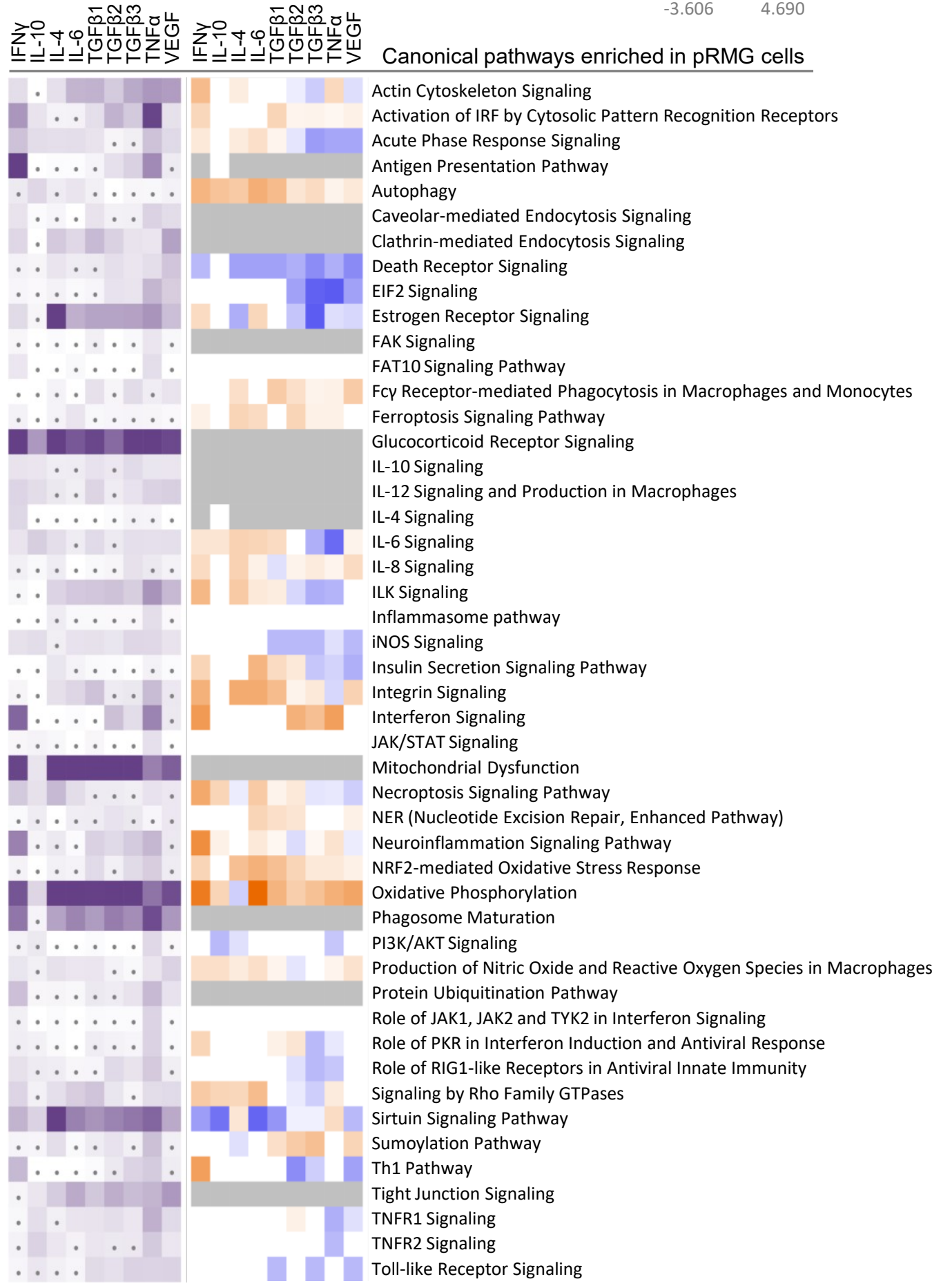

Figure 5. A comparative IPA analysis with the significantly regulated proteins identified in the pRMG lysates after stimulation with the indicated cytokines was performed. Canonical pathways related to signaling, cell death, immune system processes and oxidative stress were selected. Pathways with significant enrichment of genes after stimulation with at least one cytokine are presented. Significance of the gene enrichment for each pathway and treatment is indicated by purple squares in the left array. Thereby, treatments that did not meet the

This is a provisional file, not the final typeset article 

significance threshold ( $\mathrm{p}$-value $\leq 0.05$ ) are marked with a dot. The $\mathrm{z}$-score is indicated in the right array and represents a prediction of activation (orange) or inhibition (blue) of the pathway. Gray squares mark treatments where the activation state of a pathway could not be calculated.

Among the most significantly regulated pathways in MIO-M1 cells and pRMG were the canonical pathways "Mitochondrial Dysfunction" and "Oxidative Phosphorylation". These pathways were significantly induced by all examined cytokines in pRMG. The pathways "Ferroptosis Signaling Pathway", "iNOS Signaling", "NRF2-mediated Oxidative Stress Response", and "Production of Nitric Oxide and Reactive Oxygen Species in Macrophages" are closely linked to the cellular redox state and were among the enriched pathways in MIO-M1 cells and pRMG after treatment with various cytokines. Furthermore, proteins associated with the maturation of phagosomes were significantly enriched in pRMG. In line with this, "Caveolar-mediated Endocytosis Signaling” was significantly enriched in pRMG after treatment with IFN $\gamma$, TGF $\beta 1$, TNF $\alpha$ and VEGF, and "Clathrinmediated Endocytosis Signaling" was significantly enriched in pRMG after treatment with all cytokines except IL-10. Besides these two pathways associated with the recycling of the extracellular environment, intracellular protein homeostasis and MHC class I peptide generation was facilitated by enrichment of the "Protein Ubiquitination Pathway" in pRMG after treatment with IFN $\gamma, \mathrm{TGF} \beta 3$, TNF $\alpha$ and VEGF. Similarly, IFN $\gamma$, IL-4, TGF $\beta 1$, TGF $\beta 3$, TNF $\alpha$ and VEGF enriched "Protein Ubiquitination Signaling" in MIO-M1 cells. "Neuroinflammation Signaling" was induced by IFN $\gamma$, TNF $\alpha$ and VEGF in MIO-M1 cells and by IFN $\gamma$, TGF $\beta 1$, TGF $\beta 3$ and TNF $\alpha$ in pRMG, whereas TGF $\beta 2$ and VEGF led to a slight inhibition of this pathway in pRMG.

\subsection{A deeper look into Müller cell complement secretion upon cytokine stimulation}

Because the enrichment analysis of the secretome yielded highly significant hits such as "humoral immune response" and "immune system process," we took a closer look at complement proteins in the secretome and cell lysates. Notably, most complement proteins are secreted as key components of the humoral immune system. The identified complement components include central complement proteins, regulators, and receptors. Consistent with their localization in the cell membrane, the latter (including ITGAM, ITGB2, C5aR1) were detected only in cell lysates, and here specifically in those of pRMGs. The complement regulators clusterin (CLU), vitronectin (VTN), CD59, and SERPING were found in most test samples. With regard to the central complement components, the pRMG secretome took a prominent position and showed results for complement components for all three different activation pathways (e.g. C1q, FD, MASP1) and the terminal pathway (e.g. C9). The central complement protein $\mathrm{C} 3$ was found in both the MIO-M1 and pRMG secretomes and in the MIO-M1 lysate. Interestingly, cytokine treatment induced changes in complement proteins and regulators but had no effect on complement receptor expression. We observed that C1q subunits, which initiate the classical complement pathway by binding to antibodies, were detectable only in pRMG but not in MIO-M1 cells. C1q levels in cell lysates and the corresponding secretome were consistently reduced after TNF $\alpha$ treatment but were increased by IFN $\gamma$. Moreover, complement proteases $\mathrm{C} 1 \mathrm{r}$ and $\mathrm{C} 1 \mathrm{~s}$, which bind to $\mathrm{Clq}$ therewith continuing the cascade of classical pathway activation, were enriched in the supernatants of MIO-M1 and pRMG cells treated with IFN $\gamma$ (Fig. 2C). In contrast, C1r concentration was significantly decrease in supernatants of MIO-M1 cells but not pRMG after VEGF and TGF $\beta 2$ application. Notably, C1s and C1r were not detected in cellular lysates. Interestingly, the abundance of the central complement proteins $\mathrm{C} 3$ and $\mathrm{C} 4 \mathrm{~A}$ were modified by the supplemented cytokines in MIO-M1 secretomes only and not in any other data set (Fig. 2C). These proteins are cleaved upon complement activation as for example triggered by the C1q-mediated classical pathway and result in cleaved products which interact with cellular receptors (e.g. C3a/C3b, C4a). Here, 
417 complement protein C3 is mainly increased following TNF $\alpha$ addition and $\mathrm{C} 4$ upon exposure to IFN $\gamma$ 418 (Fig. 2C). In fact, IFN $\gamma$ was also the major player modulating the secreted complement components 419 in pRMG: C2 and FI were significantly increased while C9, FD and MASP1 were clearly reduced in 420 its presence. These complement components absent from any other sample.

421 Regarding the complement regulators factor H (FH), SERPING and CLU are of interest. Secretion of 422 FH was not observed in untreated MIO-M1 and pRMG, but it was significantly upregulated in MIO423 M1 secretomes following IFN $\gamma, \mathrm{TNF} \alpha$, TGF $\beta 3$, and VEGF treatment (Fig. 2A). Similar results were 424 obtained for SERPING, whose levels were increased by IFN $\gamma$ in the MIO-M1 secretome, pRMG cell 425 lysates and secretome. Remarkably, the MIO-M1 lysate showed decreased values for CLU following 426 IFN $\gamma$, TGF $\beta 1$ and TNF $\alpha$, and similar but not significant trends was observed for the respective secretome. Finally, while CLU was upregulated in pRMGs lysates upon IL-6 or VEGF treatment, no significant alterations could be found in corresponding secretomes.

429 In summary, IFN $\gamma$ and TNF $\alpha$ seemed to be the most effective cytokines to modulate the Müller cell 430 complement expression and secretion (Fig. 2).

\section{$431 \quad 3.5 \quad$ Müller cells as atypcial antigen-presenting cells}

432 Intriguingly, treatment of $\mathrm{pRMG}$ with IFN $\gamma, \mathrm{TGF} \beta 2$, TGF $\beta 3$ and TNF $\alpha$ significantly enriched 433 proteins associated with the "Antigen Presentation Pathway". Likewise, the "Antigen Presentation 434 Pathway" was induced in MIO-M1 cells by treatment with IFN $\gamma$, TGF $\beta 1$, TNF $\alpha$ and VEGF. Thereby, 435 antigen presentation is an umbrella term for two distinct processes. MHC class I antigen presentation 436 is common to all nucleated cells and allows $\mathrm{CD}^{+}$cytotoxic T cells (CTL) to assess whether cells are 437 infected with an intracellular pathogen $(50,51)$. In contrast, MHC class II is presented to antigen 438 specific $\mathrm{CD}^{+} \mathrm{T}$ cells mainly by professional antigen-presenting cells inducing their activation and 439 differentiation to $\mathrm{T}$ helper cells (52). 
A
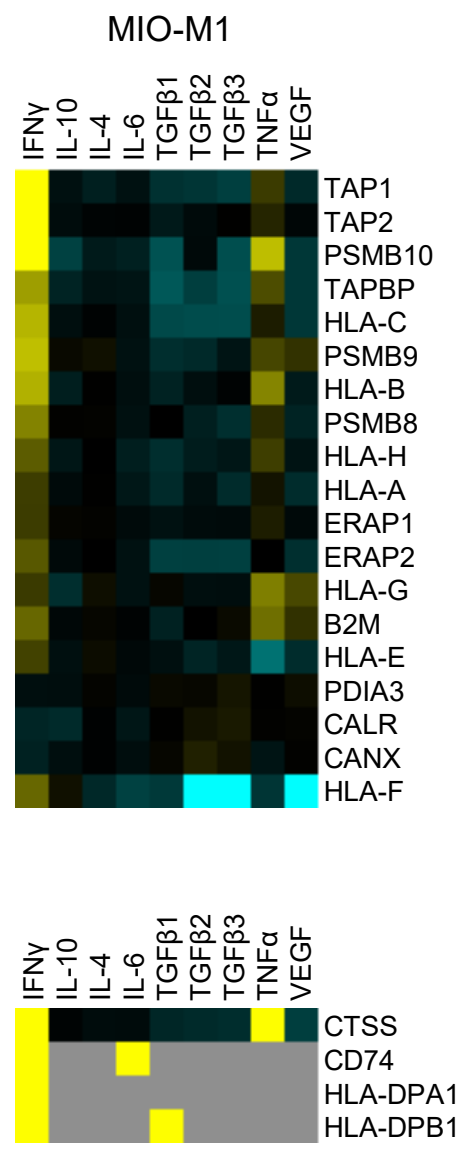

$\log 2$ ratio stimulated/ctrl

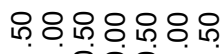

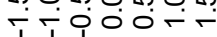

B
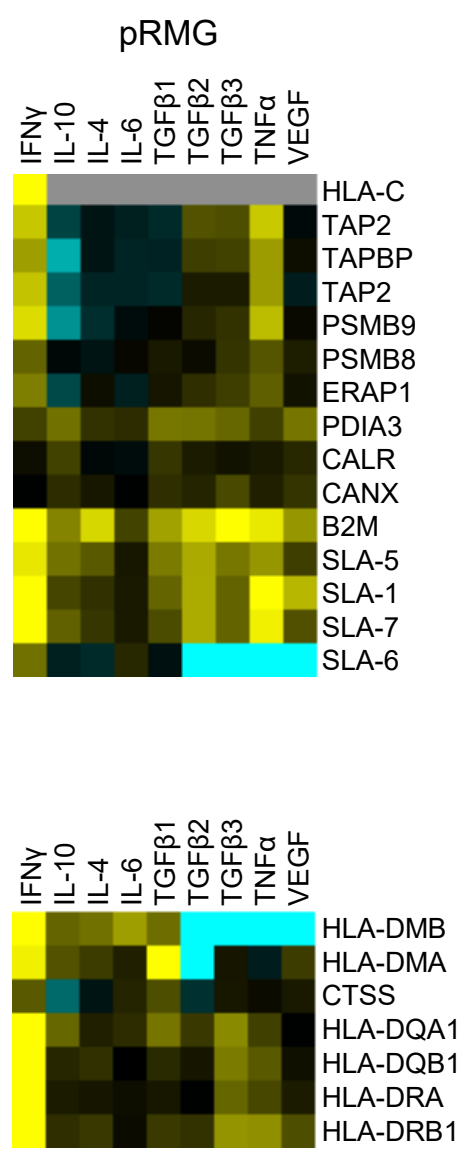

$\log 2$ ratio

stimulated/ctrl

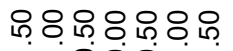

广ั

Figure 6. Heatmap of hierarchical cluster analysis of proteins involved in MHC class I (upper panel) and MHC class II (lower panel) antigen presentation expressed by MIO-M1 cells (A) and pRMG cells (B) after treatment with various cytokines. Down-regulated proteins are presented in cyan, while up-regulated proteins are depicted yellow for the respective treatments. Gray squares represent proteins that were neither identified in the untreated control, nor in the respective treatment. The heatmap was generated on the basis of the log2 fold change of the respective proteins.

To investigate the antigen presentation capacity of Müller cells, we constructed a hierarchical heatmap for MIO-M1 cells (Fig. 6A) and pRMG (Fig. 6B) challenged with various cytokines. Proteins linked to antigen presentation were selected and clustered hierarchically. Proteins associated with MHC class I antigen presentation are displayed in the upper panel and proteins correlated to MHC class II antigen presentation are depicted in the lower panel. In MIO-M1 cells, IFN $\gamma$ induced the majority of proteins linked to both, MHC class I and II antigen presentation, whereas TNF $\alpha$ exerted its inductive effect exclusively on MHC class I antigen presentation. The only protein linked to $\mathrm{MHC}$ class II antigen presentation induced by TNF $\alpha$ in MIO-M1 cells was Cathepsin S (CTSS). Furthermore, the other cytokines did not induce proteins related to antigen presentation in MIO-M1 cells. Quite the contrary, TGF $\beta 2$ and TGF $\beta 3$ reduced the abundance of proteins linked to MHC class 
I antigen presentation in these cells. In contrast to MIO-M1 cells, pRMG reacted to all tested cytokines by induction of components of both MHC class I and II antigen presentation to varying degrees. IFN $\gamma$ and TNF $\alpha$ induced proteins of MHC class I and II antigen presentation in pRMG, among others SLA-DQA1, SLA-DQB1, SLA-DRA and SLA-DRB1. Furthermore, class I and II antigen presentation was upregulated by TGF $\beta$ isoforms 1-3 in pRMG. While TGF $\beta 2$ and TGF $\beta 3$ induced the components of the MHC class I peptide loading complex TAP2 and TAPBP, TGF $\beta 1$ also increased the abundance of HLA-DMA and HLA-DMB, proteins involved in the peptide loading on MHC class II. We saw only a subtle induction of proteins related to antigen presentation by IL-10, IL-4 and IL-6. The smallest impact on antigen presentation proteins was seen after stimulation with IL-6. Furthermore, IFN $\gamma$ significantly upregulated the expression of the co-stimulatory molecule CD40 in pRMG, while TGF $\beta 2$, TGF $\beta 3$, TNF $\alpha$ and VEGF resulted in lower abundance of CD40

469 (Suppl. Table 4).

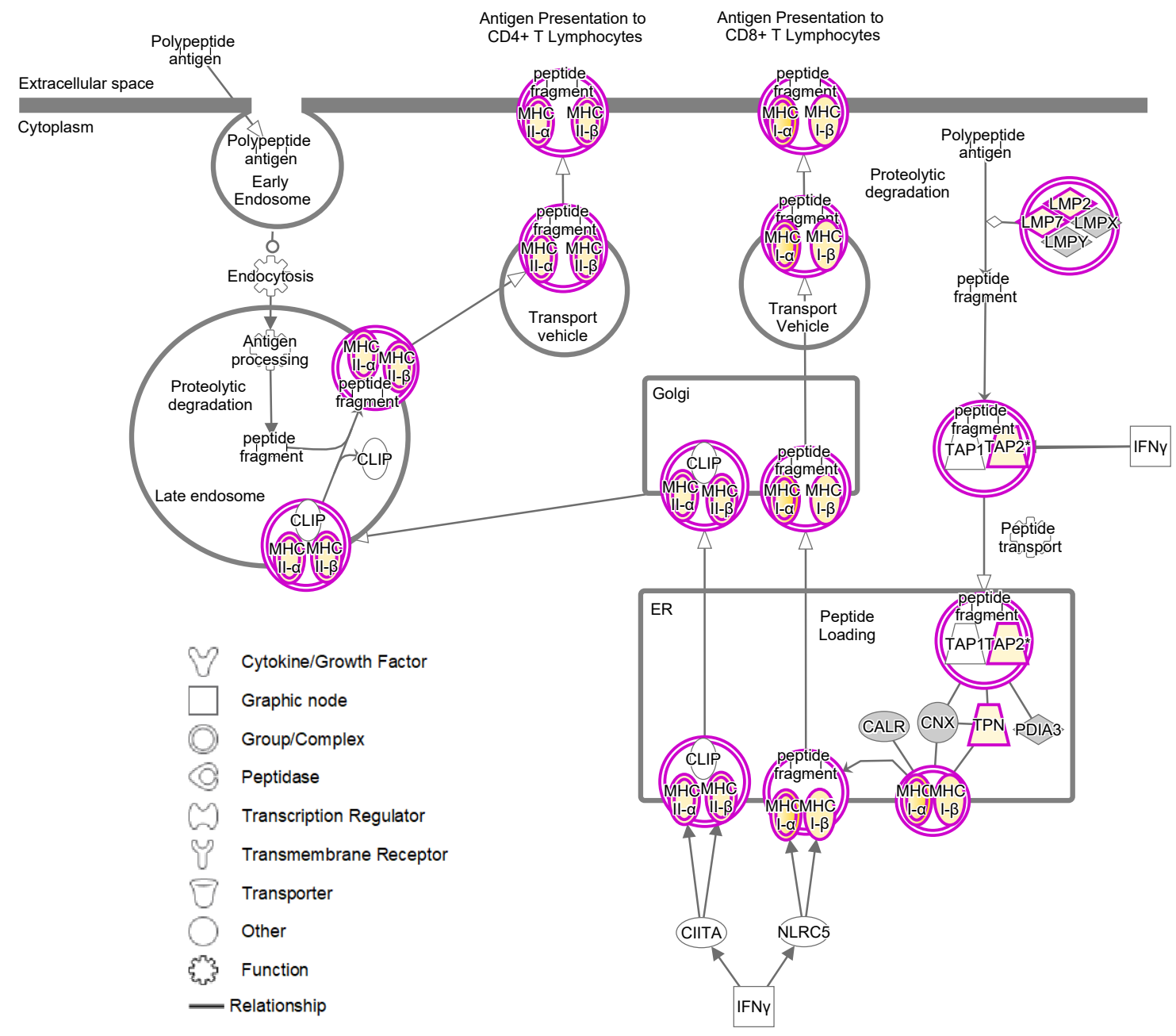

Figure 7. IPA for $\mathrm{pRMG}$ cells after treatment with IFN $\gamma$ was performed. Depicted is the yellow color indicates the degree of upregulation. Grey proteins are in the dataset but did not 

circle) or complexes (double circles).

Induction of the canonical MHC class I and MHC class II antigen presentation pathway as assessed by IPA for $\mathrm{pRMG}$ after treatment with IFN $\gamma$ is summarized in Fig. 7. This pathway was enriched in pRMG cells with a p-value of $4.15 \times 10^{-12}$. Besides MHC class I and MHC class II, also components of the peptide loading complex of MHC class I (TAP2 and TPN) were upregulated in pRMG after IFN $\gamma$ treatment. Furthermore, IFN $\gamma$ induces the Large Multifunctional Peptidase 2 (LMP2; synonymous to PSMB9) and Large Multifunctional Peptidase 7 (LMP7; synonymous to PSMB8) subunits of the immunoproteasome in MIO-M1 cells.

\section{Discussion}

DR is a condition associated with microvascular degeneration, resulting in ocular inflammation and eventually in complete blindness (53). As the prevalence of diabetes in developed and developing countries rises, DR became the leading cause of blindness in the working-age population (54). Microglia are resident innate immune cells in the retina and as such, they are considered to regulate the inflammation during DR (55). In contrast, the role of Müller cells during the pathology of DR has been often described to be the metabolic support of neurons (53). However, growing evidence suggests excessive signaling between Müller cells and the surrounding tissue including microglia, with both beneficial and detrimental consequences for the pathogenesis of DR (56). We show here that stimulation of Müller cells with pro-inflammatory cytokines like IFN $\gamma$ or TNF $\alpha$, but also with growth factors like TGF $\beta$ or VEGF resulted in profound, yet distinct response profiles in their secretomes, thus confirming their central role in cell-to-cell communication within the retina.

495

496

497

Previously, it has been shown that the conditional knock-out of VEGF in Müller cells of mice reduced the expression of inflammatory markers like TNF $\alpha$ or ICAM1 compared to diabetic control mice, indicating a pro-inflammatory effect of VEGF on Müller cells $(57,58)$. Here we could show that VEGF induced the Müller cell line MIO-M1 to secrete proteins associated with immune effector processes like IFN $\gamma, \mathrm{B} 2 \mathrm{M}, \mathrm{HRNR}$, and complement-associated proteins like SERPING1 and complement factor $\mathrm{H}(\mathrm{FH})$. Furthermore, ICAM1 was more abundant in our proteome data of MIOM1 cells and pRMG treated with VEGF compared to the respective control cells. Thus, we were able to confirm the pro-inflammatory role of VEGF in Müller cells.

503 Overall, we observed that MIO-M1 cells secreted TNF $\alpha$ upon stimulation with VEGF, IL-6, and the TGF $\beta$ isoforms 2 and 3. Increased levels of TNF $\alpha$ were previously identified in the vitreous of DR patients (59). Furthermore, Müller cells have been reported to secrete increased amounts of VEGF and TNF $\alpha$ under stress conditions like inflammation or hyperglycemia, thereby promoting retinal inflammation (57, 60-64). Our results show that TNF $\alpha$ induced MIO-M1 cells to secrete inflammatory proteins but also proteins associated with tissue development like Leukemia Inhibitory Factor (LIF), Follistation (FST), Neuregulin 1 (NRG1), or Pleiotrophin (PTN), to name but a few. Thus, our data suggest a pleotropic role of TNFa in Müller cells.

511 TGF $\beta$ s regulate early embryogenesis, maintenance and regeneration of mature tissues, and various disease processes (65). TGF $\beta 1$ has been described to be anti-inflammatory, inducing immune tolerance in the periphery and preventing autoimmunity. Thus, knock out of TGF $\beta 1$ in mice induced autoantibodies and multifocal inflammatory disease in many tissues, eventually causing the mice to die within 4 weeks of age (66). In our in vitro model, treatment of MIO-M1 cells with TGF $\beta 1$ induced secretion of CCL2 and therefore might result in the recruitment of leukocytes. 
Simultaneously, TGF $\beta 1$ enhanced the secretion of anti-inflammatory proteins like TNF Alpha

518 Induced Protein 6 (TNFAIP6) or LIF in Müller cells. In contrast, TGF $\beta 2$ and TGF $\beta 3$ also promoted immune system processes in MIO-M1 cells by inducing secretion of pro-inflammatory cytokines TNF $\alpha$, CXCL8, CXCL10 and IFN $\gamma$. It has been shown that TGF $\beta 2$ acts immunomodulatory in aqueous humor by decreasing the expression of IL-6, CXCL1, CCL2, G-CSF and IGFBP-5 (67). However, we demonstrated that treatment of Müller cells with TGF $\beta 2$ induced expression of IL-6 and CCL2, suggesting a complex and diverse role of TGF $\beta 2$ signaling in the eye. Although TGF $\beta$ isoforms are closely related (sharing $71-79 \%$ sequence identity), have similar three-dimensional structures and signal canonically through the same receptor (68), our secretome analyses provide evidence that they affect Müller cells differentially. While knock-out of the TGF $\beta$ isoforms resulted in different phenotypes, all null mutations were lethal (69-72). Replacement of the coding sequences by one of the other isoforms only partially rescued these phenotypes, implying intrinsic differences of these isoforms $(73,74)$. These differences might originate from varying flexibilities of an interfacial helix and thus modify the binding of the TGF $\beta$ isoforms to matrix proteins as suggested by Huang and colleagues (68). Latent TGF $\beta$ binds Thrombospondin 1 (THBS1), augmenting its activation (75-77). Intriguingly, in our study, all three TGF $\beta$ s elevate the abundance of THBS 1 indicating a positive feedback loop. Recently, transcriptome analysis linked the expression of TGF $\beta$ isoforms of mice to activation of different signaling cascades. While TGF $\beta 1$ and TGF $\beta 2$ evoked the non-canonical p38MAPK signaling pathway, which has been linked to gliosis, TGF $\beta 3$ induced the SMAD canonical signaling pathway for TGF $\beta$ s in mice $(78,79)$. During glaucoma, the aqueous humor contains elevated levels of TGF $\beta 2$, exceeding levels for homeostatic signaling $(77,80)$. Thereby, TGF $\beta 2$ has been associated with pathological remodeling of the trabecular meshwork and the optical nerve head (77). Furthermore, it stimulated secretion of extracellular matrix proteins by astrocytes and cells of the lamina cribrosa (81-84). Our data suggest that Müller cells also contribute to the remodeling of the extracellular matrix as stimulation with TGF $\beta 1$ and TGF $\beta 3$ resulted in enhanced secretion of extracellular matrix proteins like Fibrillin-2 (FBN2), various keratins, and collagens. Furthermore, they simultaneously enhanced the turnover of extracellular matrix by inducing the secretion of the Matrix Metalloprotease-1 (MMP1) and MMP2. Previously, MMP2 has been described to be elevated in DR patients and to promote the pathogenesis of DR by inducing mitochondrial dysfunction and apoptosis of retinal capillary cells (85). Intriguingly, our IPA also indicates that the canonical pathway for mitochondrial dysfunction is enriched in Müller cells upon stimulation with all TGF $\beta$ isoforms. However, this is not solely due to the enhanced secretion of MMP2, since mitochondrial dysfunction is enriched in Müller cells by all tested cytokines, whereas TGF $\beta$ s exclusively induce MMP2.

A meta-analysis of eleven studies recently showed significantly increased levels of the antiinflammatory cytokine IL-10 in the vitreous humor of DR patients (86). Moreover, IL-10 levels were higher in patients with proliferative DR compared to patients with non-proliferative DR (86). While our data show no contribution of Müller cells to elevated IL-10 secretion upon stimulation with the tested cytokines, IL-10 induced proteins associated with tissue development, cell adhesion, and angiogenesis in MIO-M1 cells, namely Corneodesmosin (CDSN), LIF, PTN, and various collagens, to name a few. In line with this, it has been shown that IL-10 is involved in the pathological angiogenesis during the development by modulating the macrophage response to hypoxia (87). Thus, we hypothesize that IL-10 might also be involved in the abnormal angiogenesis during DR. and chemokine production in macrophages following a pro-inflammatory stimulus (88-90). In line with this, we observed increased abundances of many pro-inflammatory proteins like IFN $\gamma$, S100A9, S100A7, CXCL10, and Lysozyme C (LYZ) upon stimulation of MIO-M1 cells with IL-4, indicating 
that IL-4 does not exert an anti- but a pro-inflammatory influence in these cells. Interestingly, MIOM1 cells did not secrete the anti-inflammatory interleukin IL-4 upon treatment with the various tested cytokines. Secretion of pro-inflammatory IL-6 by Müller cells has been described upon treatment with IL-1 $\beta$ or LPS (91). Furthermore, elevated levels of IL-6 were found in the vitreous and serum of DR patients and even further increased in patients suffering from proliferative DR (92). Here we show that IFN $\gamma$, TNF $\alpha$, TGF $\beta 2$, and TGF $\beta 3$ also induced the secretion of IL-6 in MIO-M1 cells. Previously, it was shown that IL1 $\beta$ induced IL-6 through activation of the p38MAPK signaling pathway (93). The murine TGF $\beta$ isoforms 1 and 2 also activated the p38MAPK signaling in Müller cells of mice (78). Thus, the involvement of p38MAPK signaling in secretion of IL-6 by Müller cells should be addressed in further studies. Brandon and colleagues demonstrated induction of VEGF by IL-6 in Müller cells, especially under hyperglycemic conditions, preventing Müller cells from glucose toxicity (94). In contrast, our analysis revealed no VEGF secretion by MIO-M1 cells upon IL-6 treatment. High glucose concentrations of $25 \mathrm{mM}$ potentiated the induction of VEGF by IL-6 (94). However, by default the standard culture medium used in our study has a D-glucose concentration of $25 \mathrm{mM}$. Thus, an adaption of MIO-M1 cells to these conditions might have occurred negating the induction of VEGF by IL-6.

Elevated levels of IFN $\gamma$ in the vitreous of DR patients have been described previously $(95,96)$. In our study, we could observe distinctly elevated INF $\gamma$ secretion only after stimulation with INF $\gamma$.

582 Treatment with IFN $\gamma$ furthermore induced the secretion of CXCL9, CXCL10, IL-6 and complement 583 subcomponent C1R in MIO-M1 cells and pRMG.

Interestingly, we observed an induction of CX3CL1 in the whole-cell lysates and in the secretomes. Specifically, except for TGF $\beta 1$ and TGF $\beta 2$, all stimulants used in this study induced expression of CX3CL1 in the cell proteome of MIO-M1 cells, while only IFN $\gamma$ and TNF $\alpha$ treatment resulted in significantly higher abundance of CX3CL1 in the secretome as well. CX3CL1 is a membrane-bound chemokine, which functions as an adhesion molecule for leukocytes, but can also be proteolytically cleaved, resulting in a soluble form with chemotactic function $(97,98)$. Several studies already demonstrated the implication of CX3CL1 in DR pathogenesis as the vitreous of PDR patients contains elevated levels of CX3CL1 (99) Furthermore, circulating CD11b+ leukocytes that are involved in leukostasis in DR express higher levels of CX3CR1 in diabetic mice compared to controls (100). Thus, membrane-bound CX3CL1 on the surface of Müller cells might be involved in leukostasis in DR. Since TNFa levels are elevated in the eyes of DR patients, this might result in the upregulation of CX3CL1 in diabetic Müller cells (101). In a previous study, incubation of microglia with Müller cell supernatant containing secreted CX3CL1 resulted in the upregulation of the respective receptor CX3CR1 in microglia. The authors proposed that Müller cells might be able to promote microglial motility via the chemotactic effect of CX3CL1 (102). Thus, secretion of CX3CL1 from Müller cells might contribute to DR pathogenesis by recruiting peripheral inflammatory cells and microglia. Upregulation of CX3CL1 after treatment with TNF $\alpha$ has been demonstrated in aortic endothelial cells, but could not be observed in retinal endothelial cells (103). Here, we demonstrate that stimulation with TNF $\alpha$ elicits secretion of CX3CL1 in pRMG and MIO-M1 cells and expression of CX3CL1 in MIO-M1 cells. Thus, we lay the ground for further research concerning the expression and regulation of CX3CL1 in Müller cells during DR.

Another route of communication between microglia/macrophages and Müller cells could be local changes in complement expression. We recently demonstrated, that Müller cells in the mouse retina are the main producers of complement components of the classical (C1s, C4), the alternative pathway (FB) and $\mathrm{C} 3$ as the central component to all pathways under homeostatic, but also under ischemic stress conditions (104). In the retina, it is the microglia that by far express highest levels of 
complement receptors including ITGAM (alias CD11b), C3aR, C5aR1 and C5aR2 (104). In our

611 present study, TNF $\alpha$ and INF $\gamma$ triggerd the most prominent effects on complement expression

612 consistently in MIO-M1 and pRMG. Given that in vivo microglia and potentially other immune cell

613 serve as major source of TNF $\alpha$ and INFy (105) (resource data from Cowan et al., 2020 (106) at https://data.iob.ch), the strong effect of these cytokines on Müller cell could be central to coordinate the tissue immune homeostasis in pathologies. In this context, the enhanced expression of activating complement components by Müller glia could serve as feedback mechanisms towards microglia in turn modulating their activation profile. Taken together, our results point towards a pro-inflammatory phenotype of Müller cells, which is in line with a previous study, where we analyzed the surfaceome of primary equine Müller cells and MIO-M1 cells after stimulation with Lipopolysaccharide (30). While the surfaceome of Müller cells in this previous study revealed expression of MHC class I and II as well as costimulatory molecules especially in primary equine Müller cells, we could now further complement these results with LC-MS/MS-analyses of whole cell lysates and of cell supernatants, confirming an antigen-presenting phenotype of Müller cells. While stimulation with LPS resulted in enhanced expression of MHC class II molecules in primary equine Müller cells, no MHC class II molecules could be identified in MIO-M1 cells upon LPS treatment (30). In contrast, stimulation with IFN $\gamma$ in this study induced the expression of proteins that are associated with both MHC-I and MHC-II antigen presentation in pRMG as well as in MIO-M1 cells. This is in accordance with an early study that demonstrated the induction of MHC class I and MHC class II molecules in primary human Müller cells by IFN $\gamma$ in vitro (107). In contrast to MIO-M1 cells, pRMG also showed basal expression of MHC class II without stimulation. Thus, our data demonstrate that Müller cells exhibit several criteria for atypical antigen-presenting cells (108). While microglial cells are the predominant immune cells of the retina, our data confirm that Müller cells are crucially involved in immunological processes in the retina as well, as they possess an antigen processing and presenting machinery and secrete pro-inflammatory cytokines (14). We have previously shown that the cultivation of primary porcine Müller cells under hyperglycemic conditions resulted in higher expression levels of MHC class II molecules, pointing towards an immunologically activated state of Müller cells in DR (36).

637 Besides expression of MHC class I and II molecules, we demonstrated that stimulation of Müller 638 cells with various cytokines resulted in the enrichment of proteins and pathways that are associated with the formation and maturation of phagosomes. Previously, Müller cells have been described to be phagocytic cells, capable of phagocytosing cell debris, dead photoreceptor cells and even bacteria (109-111). Our IPA showed that proteins of phagocytosis pathways in Müller cells are induced upon stimulation with various cytokines. Furthermore, phagocytosis is not only clathrin- but also caveolarmediated. Since our data showed enrichment of phagocytic pathways, as well as the canonical antigen presentation pathway, it is possible that Müller cells present exogenous peptides on MHC class II to CD4+ T helper cells. Intriguingly, phagocytosis of dead photoreceptors would also allow Müller cells to present proteins expressed by photoreceptors on MHC class II, and via crosspresentation on MHC class I $(112,113)$. The relationship of HLA alleles and haplotypes to the progression of diabetic retinopathy is part of an ongoing scientific debate (114-122). However, the rigorous pro-inflammatory signaling of Müller cells paired to potential activation of CD4+ and CD8+ $\mathrm{T}$ cells in the context of DR should be elaborated in further studies. Towards this goal it should be addressed, whether Müller cells are sufficient to stimulate alloreactive naïve $\mathrm{T}$ cells or memory $\mathrm{T}$ 652 cells (108).

653 Furthermore, our proteomic analysis revealed significantly higher abundance of the costimulatory molecule CD40 in pRMG after stimulation with IFN $\gamma$. Intriguingly, CD40 has been shown to play a vital role in DR pathogenesis (123-125). A study with transgenic mice, which express CD40 only in Müller cells, demonstrated that the upregulation of CD40 in Müller cells under diabetic conditions is 
sufficient for thriving leukostasis and degeneration of capillaries, thus promoting retinal inflammation and neurodegeneration in streptozotocin induced DR (124). Expression of CD40 has also been demonstrated in primary human Müller cells and its ligation with CD154 resulted in increased expression of ICAM-1 in Müller cells (123). Furthermore, it was shown that activation of Müller cells through CD40 leads to an enhanced production of TNF $\alpha$ and IL-1 $\beta$ in bystander microglia (124). Since several studies revealed an association between elevated levels of IFN $\gamma$ and DR, and stimulation of CNS astrocytes led to expression of CD40, IFN $\gamma$ in DR might lead to the upregulation of CD40 in Müller cells and thus promote chronic inflammation (126-129). Here, we provide new insight in CD40 signaling in Müller cells, as IFN $\gamma$ increased the expression of CD40, whereas TGF $\beta 2$, TGF $\beta 3$, TNF $\alpha$ and VEGF resulted in lower abundance of CD40 in pRMG. Future studies are needed to further investigate this issue, as the CD40 signaling pathway constitutes a possibility for therapeutical intervention (130). In contrast to the porcine dataset, we could not identify CD40 in the MIO-M1 cells. As CD40 expression has already been shown in primary human Müller cells, our result might be due to the dedifferentiation of immortalized cells in culture (123).

Oxidative stress and reactive oxygen species (ROS) are known to play a central role during the pathogenesis of DR (131). Rat-derived Müller cells under hyperglycemic conditions developed mitochondrial dysfunction and oxidative stress, causing swelling and eventually apoptosis of the cells $(132,133)$. Mitochondrial dysfunction can lead to ROS production, which then promotes inflammatory response by activation of NF- $\mathrm{B}$ and release of pro-inflammatory cytokines $(134,135)$. Our analysis revealed that proteins associated with mitochondrial dysfunction were enriched after treatment of pRMG with all tested cytokines. Furthermore, two significantly enriched pathways in our data sets are associated with reactive oxygen species, namely "NRF2 mediated Oxidative Stress Response" and "Production of Nitric Oxide and Reactive Oxygen Species in Macrophages". Intriguingly, Müller cells have previously been found to regulate the ROS levels via Nrf2 and to be more resistant to ROS formation compared to photoreceptor cells or bipolar cells $(41,136)$. In line with this, we showed that treatment with IL-4, TGF $\beta 2$, TGF $\beta 3$, TNF $\alpha$ and VEGF inhibited death receptor signaling $\mathrm{pRMG}$. Phagocytic cells often produce ROS to protect themselves from pathogens (137). Furthermore, macrophages stabilize cytosolic Nrf2 to be more resistant against ROS (138). Since Müller cells have been shown to by phagocytic, we propose that induction of ROS in these cells also serves as a defense mechanism (109-111).

In this study, we used both primary porcine Müller cells and the spontaneously immortalized human Müller cell line MIO-M1 that was established and described in the Moorfields Institute of Ophthalmology 2002 (39). Although MIO-M1 cells largely retain the phenotypic and functional characteristics of Müller cells in vitro, previous studies documented some characteristics of neuronal stem cells and expression of postmitotic neuronal cell markers in MIO-M1 cells $(39,139,140)$.

692 Furthermore, it has been shown that Müller cells tend to adapt to cell culture conditions by reduced secretion of neurotrophic factors (141-143). Our analysis revealed many proteins commonly regulated in pRMG and MIO-M1 cells, as well as pathways similarly enriched and regulated in both cells. However, we also observed effects specific for either MIO-M1 cells or pRMG. Thus, for example our IPA revealed that MIO-M1 cells decrease the activity of the significantly enriched canonical pathway oxidative phosphorylation, while it is amongst the most induced pathways of pRMG following treatment with the majority of tested cytokines. While these differences might originate from different adaption of MIO-M1 cells and pRMG to the cell culture conditions or the immortalization of MIO-M1 cells, it might as well be possible that they originate from differences in human and porcine Müller cells. Further studies should elaborate similarities and differences between freshly prepared primary human and porcine Müller cells. 
703

704

705

706

707

708

709

710

711

712

713

714

715

716

717

718

719

720

721

722

723

724

725

726

727

728

729

Taken together, our in-depth proteomic profiling of MIO-M1 cells and pRMG revealed the capacity of Müller cells to react in a differentiated manner upon treatment with various growth factors and cytokines. Furthermore, we demonstrated a primarily pro-inflammatory phenotype of Müller cells, as they secreted a variety of pro-inflammatory cytokines, complement components and upregulated proteins associated with antigen processing and presentation, suggesting a function as atypical antigen-presenting cell in the course of retinal inflammation. Furthermore, we observed enrichment of proteins connected to mitochondrial dysfunction, as well as proteins related to the formation and maturation of phagosomes. Thus, Müller cells are capable of modulating immune responses in the retina, and may significantly contribute to chronic inflammation during DR.

\section{Conflict of Interest}

The authors declare that the research was conducted in the absence of any commercial or financial relationships that could be construed as a potential conflict of interest.

\section{Author Contributions}

Conceptualization, S.M.H. and C.A.D.; Formal analysis, S.M.H.; Funding acquisition, S.M.H. and C.A.D.; Investigation, A.S., L.L., C.A.D., and S.M.H.; Project administration, S.M.H.; Supervision, S.M.H.; Visualization, A.S.; Writing - original draft, A.S., L.L., A.G., and D.P.; Writing - review and editing, C.A.D. and S.M.H. All authors have read and agreed to the published version of the manuscript.

\section{$7 \quad$ Funding}

This work was funded by Deutsche Forschungsgemeinschaft in the SPP 2127, grant numbers DFG DE 719/7-1 to C.A.D., HA 6014/5-1 to S.M.H., GR 4403/5-1 to A.G. and PA 1844/3-1 to D.P.

\section{Acknowledgments}

The authors would like to thank Adrian Sandbiller for providing porcine eye samples as well as Juliane Merl-Pham for critical discussions.

\section{Data Availability Statement}

All data are contained within the article or in supplementary materials. 


\section{References}

731 1. Duncan JL, Pierce EA, Laster AM, Daiger SP, Birch DG, Ash JD, et al. Inherited Retinal

732 Degenerations: Current Landscape and Knowledge Gaps. Transl Vis Sci Technol. 2018;7(4):6.

733 2. Wooff Y, Man SM, Aggio-Bruce R, Natoli R, Fernando N. IL-1 Family Members Mediate

734 Cell Death, Inflammation and Angiogenesis in Retinal Degenerative Diseases. Front Immunol.

$735 \quad 2019 ; 10: 1618$.

736 3. Olivares-González L, Velasco S, Campillo I, Rodrigo R. Retinal Inflammation, Cell Death

737 and Inherited Retinal Dystrophies. Int J Mol Sci. 2021;22(4).

738 4. Forrester JV, Kuffova L, Delibegovic M. The Role of Inflammation in Diabetic Retinopathy. 739 Front Immunol. 2020;11:583687.

740 5. Broadgate S, Yu J, Downes SM, Halford S. Unravelling the genetics of inherited retinal

741 dystrophies: Past, present and future. Prog Retin Eye Res. 2017;59:53-96.

742 6. Lechner J, O'Leary OE, Stitt AW. The pathology associated with diabetic retinopathy. Vision 743 Research. 2017;139:7-14.

$7447 . \quad$ Collaborators GBaVI. Causes of blindness and vision impairment in 2020 and trends over 30

745 years, and prevalence of avoidable blindness in relation to VISION 2020: the Right to Sight: an

746 analysis for the Global Burden of Disease Study. Lancet Glob Health. 2021;9(2):e144-e60.

747 8. Chen M, Obasanmi G, Armstrong D, Lavery NJ, Kissenpfennig A, Lois N, et al. STAT3 748 activation in circulating myeloid-derived cells contributes to retinal microvascular dysfunction in 749 diabetes. J Neuroinflammation. 2019;16(1):138.

750 9. Gerhardinger C, Costa MB, Coulombe MC, Toth I, Hoehn T, Grosu P. Expression of acute751 phase response proteins in retinal Müller cells in diabetes. Invest Ophthalmol Vis Sci.

752 2005;46(1):349-57.

753 10. Mizutani M, Gerhardinger C, Lorenzi M. Müller cell changes in human diabetic retinopathy. 754 Diabetes. 1998;47(3):445-9.

755 11. Zeng HY, Green WR, Tso MO. Microglial activation in human diabetic retinopathy. Arch

756 Ophthalmol. 2008;126(2):227-32.

757 12. Coughlin BA, Feenstra DJ, Mohr S. Müller cells and diabetic retinopathy. Vision Research. $758 \quad 2017 ; 139: 93-100$.

759 13. Kinuthia UM, Wolf A, Langmann T. Microglia and Inflammatory Responses in Diabetic 760 Retinopathy. Front Immunol. 2020;11:564077.

761 14. Karlstetter M, Scholz R, Rutar M, Wong WT, Provis JM, Langmann T. Retinal microglia:

762 just bystander or target for therapy? Prog Retin Eye Res. 2015;45:30-57.

763 15. Di Pierdomenico J, Martínez-Vacas A, Hernández-Muñoz D, Gómez-Ramírez AM, Valiente-

764 Soriano FJ, Agudo-Barriuso M, et al. Coordinated Intervention of Microglial and Müller Cells in 765 Light-Induced Retinal Degeneration. Invest Ophthalmol Vis Sci. 2020;61(3):47.

766 16. Wang M, Ma W, Zhao L, Fariss RN, Wong WT. Adaptive Müller cell responses to microglial 767 activation mediate neuroprotection and coordinate inflammation in the retina. J Neuroinflammation. $7682011 ; 8: 173$.

769 17. Reichenbach A, Bringmann A. Glia of the human retina. Glia. 2020;68(4):768-96. 
18. Newman E, Reichenbach A. The Müller cell: a functional element of the retina. Trends Neurosci. 1996;19(8):307-12.

772 19. Bringmann A, Pannicke T, Grosche J, Francke M, Wiedemann P, Skatchkov SN, et al. Müller cells in the healthy and diseased retina. Prog Retin Eye Res. 2006;25(4):397-424. signaling and factors involved in Müller cell gliosis: Neuroprotective and detrimental effects. Prog

776 Retin Eye Res. 2009;28(6):423-51.

22. von Toerne C, Menzler J, Ly A, Senninger N, Ueffing M, Hauck SM. Identification of a

24. Subirada PV, Paz MC, Ridano ME, Lorenc VE, Vaglienti MV, Barcelona PF, et al. A journey into the retina: Müller glia commanding survival and death. European Journal of Neuroscience. 2018;47(12):1429-43.

792 26. Natoli R, Fernando N, Madigan M, Chu-Tan JA, Valter K, Provis J, et al. Microglia-derived

793 IL-1 $\beta$ promotes chemokine expression by Müller cells and RPE in focal retinal degeneration. Mol

794 Neurodegener. 2017;12(1):31.

27. Rutar M, Natoli R, Chia RX, Valter K, Provis JM. Chemokine-mediated inflammation in the degenerating retina is coordinated by Müller cells, activated microglia, and retinal pigment epithelium. J Neuroinflammation. 2015;12(1):8.

28. Roberge FG, Caspi RR, Nussenblatt RB. Glial retinal Müller cells produce IL-1 activity and have a dual effect on autoimmune T helper lymphocytes. Antigen presentation manifested after removal of suppressive activity. J Immunol. 1988;140(7):2193-6.

803 30. Lorenz L, Hirmer S, Schmalen A, Hauck SM, Deeg CA. Cell Surface Profiling of Retinal

804 Muller Glial Cells Reveals Association to Immune Pathways after LPS Stimulation. Cells.

805 2021;10(3).

806 31. Middleton S. Porcine ophthalmology. Vet Clin North Am Food Anim Pract. 2010;26(3):55780772.

808 32. Kleinwort KJH, Amann B, Hauck SM, Hirmer S, Blutke A, Renner S, et al. Retinopathy with 809 central oedema in an INS (C94Y) transgenic pig model of long-term diabetes. Diabetologia.

$810 \quad 2017 ; 60(8): 1541-9$. 
811 33. Renner S, Blutke A, Clauss S, Deeg CA, Kemter E, Merkus D, et al. Porcine models for

812 studying complications and organ crosstalk in diabetes mellitus. Cell and Tissue Research. 2020.

813 34. Konigsberg IR, Borie R, Walts AD, Cardwell J, Rojas M, Metzger F, et al. Molecular 814 Signatures of Idiopathic Pulmonary Fibrosis. Am J Respir Cell Mol Biol. 2021.

815 35. Weigand M, Hauck SM, Deeg CA, Degroote RL. Deviant proteome profile of equine 816 granulocytes associates to latent activation status in organ specific autoimmune disease. $\mathrm{J}$

817 Proteomics. 2021;230:103989.

818 36. Sagmeister S, Merl-Pham J, Petrera A, Deeg CA, Hauck SM. High glucose treatment 819 promotes extracellular matrix proteome remodeling in Mller glial cells. PeerJ. 2021;9:e11316.

820 37. Eberhardt C, Amann B, Stangassinger M, Hauck SM, Deeg CA. Isolation, characterization 821 and establishment of an equine retinal glial cell line: a prerequisite to investigate the physiological

822 function of Muller cells in the retina. J Anim Physiol Anim Nutr (Berl). 2012;96(2):260-9.

823 38. Hauck SM, Suppmann S, Ueffing M. Proteomic profiling of primary retinal Muller glia cells 824 reveals a shift in expression patterns upon adaptation to in vitro conditions. Glia. 2003;44(3):251-63.

825 39. Limb GA, Salt TE, Munro PM, Moss SE, Khaw PT. In vitro characterization of a 826 spontaneously immortalized human Muller cell line (MIO-M1). Invest Ophthalmol Vis Sci. 827 2002;43(3):864-9.

828 40. Wisniewski JR, Zougman A, Nagaraj N, Mann M. Universal sample preparation method for 829 proteome analysis. Nat Methods. 2009;6(5):359-62.

830 41. Grosche A, Hauser A, Lepper MF, Mayo R, von Toerne C, Merl-Pham J, et al. The Proteome 831 of Native Adult Muller Glial Cells From Murine Retina. Mol Cell Proteomics. 2016;15(2):462-80.

832 42. Kall L, Canterbury JD, Weston J, Noble WS, MacCoss MJ. Semi-supervised learning for 833 peptide identification from shotgun proteomics datasets. Nat Methods. 2007;4(11):923-5.

834 43. Navarro P, Trevisan-Herraz M, Bonzon-Kulichenko E, Nunez E, Martinez-Acedo P, Perez835 Hernandez D, et al. General statistical framework for quantitative proteomics by stable isotope 836 labeling. J Proteome Res. 2014;13(3):1234-47.

837 44. Benjamini Y, Drai D, Elmer G, Kafkafi N, Golani I. Controlling the false discovery rate in 838 behavior genetics research. Behav Brain Res. 2001;125(1-2):279-84.

839 45. Kramer A, Green J, Pollard J, Jr., Tugendreich S. Causal analysis approaches in Ingenuity 840 Pathway Analysis. Bioinformatics. 2014;30(4):523-30.

841 46. Fisher RA. On the Interpretation of $\chi^{2}$ from Contingency Tables, and the Calculation of $\mathrm{P}$. 842 Journal of the Royal Statistical Society. 1922;85(1):87-94.

843 47. Eisen MB, Spellman PT, Brown PO, Botstein D. Cluster analysis and display of genome844 wide expression patterns. Proc Natl Acad Sci U S A. 1998;95(25):14863-8.

845 48. Saldanha AJ. Java Treeview--extensible visualization of microarray data. Bioinformatics. $846 \quad 2004 ; 20(17): 3246-8$.

847 49. Lunney JK, Ho CS, Wysocki M, Smith DM. Molecular genetics of the swine major 848 histocompatibility complex, the SLA complex. Dev Comp Immunol. 2009;33(3):362-74.

849 50. Sigal LJ. Activation of CD8 T Lymphocytes during Viral Infections. Encyclopedia of 850 Immunobiology. 2016:286-90. 
851

852

853

854

855

856

857

858

859

860

861

862

863

864

865

866

867

868

869

870

871

872

873

874

875

876

877

878

879

880

881

882

883

884

885

886

887

888

889

890

51. Hewitt EW. The MHC class I antigen presentation pathway: strategies for viral immune evasion. Immunology. 2003;110(2):163-9.

52. Roche PA, Furuta $\mathrm{K}$. The ins and outs of MHC class II-mediated antigen processing and presentation. Nat Rev Immunol. 2015;15(4):203-16.

53. Lechner J, O'Leary OE, Stitt AW. The pathology associated with diabetic retinopathy. Vision Res. 2017;139:7-14.

54. Zheng Y, He M, Congdon N. The worldwide epidemic of diabetic retinopathy. Indian J Ophthalmol. 2012;60(5):428-31.

55. Ginhoux F, Greter M, Leboeuf M, Nandi S, See P, Gokhan S, et al. Fate mapping analysis reveals that adult microglia derive from primitive macrophages. Science. 2010;330(6005):841-5.

56. Coughlin BA, Feenstra DJ, Mohr S. Muller cells and diabetic retinopathy. Vision Res. 2017;139:93-100.

57. Wang J, Xu X, Elliott MH, Zhu M, Le YZ. Muller cell-derived VEGF is essential for diabetes-induced retinal inflammation and vascular leakage. Diabetes. 2010;59(9):2297-305.

58. Bai Y, Ma JX, Guo J, Wang J, Zhu M, Chen Y, et al. Muller cell-derived VEGF is a significant contributor to retinal neovascularization. J Pathol. 2009;219(4):446-54.

59. Demircan N, Safran BG, Soylu M, Ozcan AA, Sizmaz S. Determination of vitreous interleukin-1 (IL-1) and tumour necrosis factor (TNF) levels in proliferative diabetic retinopathy. Eye (Lond). 2006;20(12):1366-9.

60. Kelly K, Wang JJ, Zhang SX. The unfolded protein response signaling and retinal Muller cell metabolism. Neural Regen Res. 2018;13(11):1861-70.

61. Zhong Y, Li J, Chen Y, Wang JJ, Ratan R, Zhang SX. Activation of endoplasmic reticulum stress by hyperglycemia is essential for Muller cell-derived inflammatory cytokine production in diabetes. Diabetes. 2012;61(2):492-504.

62. Zhang SX, Wang JJ, Gao G, Shao C, Mott R, Ma JX. Pigment epithelium-derived factor (PEDF) is an endogenous antiinflammatory factor. FASEB J. 2006;20(2):323-5.

63. He J, Wang H, Liu Y, Li W, Kim D, Huang H. Blockade of vascular endothelial growth factor receptor 1 prevents inflammation and vascular leakage in diabetic retinopathy. J Ophthalmol. 2015;2015:605946.

64. Liu Y, Li L, Pan N, Gu J, Qiu Z, Cao G, et al. TNF-alpha released from retinal Muller cells aggravates retinal pigment epithelium cell apoptosis by upregulating mitophagy during diabetic retinopathy. Biochem Biophys Res Commun. 2021;561:143-50.

65. Gilbert RWD, Vickaryous MK, Viloria-Petit AM. Signalling by Transforming Growth Factor Beta Isoforms in Wound Healing and Tissue Regeneration. J Dev Biol. 2016;4(2).

66. Yaswen L, Kulkarni AB, Fredrickson T, Mittleman B, Schiffman R, Payne S, et al. Autoimmune manifestations in the transforming growth factor-beta 1 knockout mouse. Blood. 1996;87(4):1439-45.

67. Yamagami S, Yokoo S, Mimura T, Amano S. Effects of TGF-beta2 on immune responserelated gene expression profiles in the human corneal endothelium. Invest Ophthalmol Vis Sci. 2004;45(2):515-21.

This is a provisional file, not the final typeset article 
68. Huang T, Schor SL, Hinck AP. Biological activity differences between TGF-betal and TGFbeta3 correlate with differences in the rigidity and arrangement of their component monomers. Biochemistry. 2014;53(36):5737-49.

894 69. Kulkarni AB, Huh CG, Becker D, Geiser A, Lyght M, Flanders KC, et al. Transforming 895 growth factor beta 1 null mutation in mice causes excessive inflammatory response and early death. 896 Proc Natl Acad Sci U S A. 1993;90(2):770-4. of the mouse transforming growth factor-beta 1 gene results in multifocal inflammatory disease. Nature. 1992;359(6397):693-9.

900 71. Sanford LP, Ormsby I, Gittenberger-de Groot AC, Sariola H, Friedman R, Boivin GP, et al. 901 TGFbeta2 knockout mice have multiple developmental defects that are non-overlapping with other 902 TGFbeta knockout phenotypes. Development. 1997;124(13):2659-70.

903 72. Proetzel G, Pawlowski SA, Wiles MV, Yin M, Boivin GP, Howles PN, et al. Transforming 904 growth factor-beta 3 is required for secondary palate fusion. Nat Genet. 1995;11(4):409-14.

905 73. Yang LT, Kaartinen V. Tgfb1 expressed in the Tgfb3 locus partially rescues the cleft palate 906 phenotype of Tgfb3 null mutants. Dev Biol. 2007;312(1):384-95.

907 74. Hall BE, Wankhade UD, Konkel JE, Cherukuri K, Nagineni CN, Flanders KC, et al.

908 Transforming growth factor-beta3 (TGF-beta3) knock-in ameliorates inflammation due to TGF-beta1 909 deficiency while promoting glucose tolerance. J Biol Chem. 2013;288(44):32074-92.

910 75. Schultz-Cherry S, Lawler J, Murphy-Ullrich JE. The type 1 repeats of thrombospondin 1

911 activate latent transforming growth factor-beta. J Biol Chem. 1994;269(43):26783-8.

912 76. Schultz-Cherry S, Murphy-Ullrich JE. Thrombospondin causes activation of latent

913 transforming growth factor-beta secreted by endothelial cells by a novel mechanism. J Cell Biol.

914 1993;122(4):923-32.

915 77. Murphy-Ullrich JE, Downs JC. The Thrombospondin1-TGF-beta Pathway and Glaucoma. J

916 Ocul Pharmacol Ther. 2015;31(7):371-5.

917 78. Conedera FM, Quintela Pousa AM, Presby DM, Mercader N, Enzmann V, Tschopp M.

918 Diverse Signaling by TGFbeta Isoforms in Response to Focal Injury is Associated with Either

919 Retinal Regeneration or Reactive Gliosis. Cell Mol Neurobiol. 2021;41(1):43-62.

920 79. Kaminska B, Gozdz A, Zawadzka M, Ellert-Miklaszewska A, Lipko M. MAPK signal 921 transduction underlying brain inflammation and gliosis as therapeutic target. Anat Rec (Hoboken). 922 2009;292(12):1902-13.

923 80. Tripathi RC, Li J, Chan WF, Tripathi BJ. Aqueous humor in glaucomatous eyes contains an 924 increased level of TGF-beta 2. Exp Eye Res. 1994;59(6):723-7.

925 81. Fuchshofer R, Birke M, Welge-Lussen U, Kook D, Lutjen-Drecoll E. Transforming growth 926 factor-beta 2 modulated extracellular matrix component expression in cultured human optic nerve 927 head astrocytes. Invest Ophthalmol Vis Sci. 2005;46(2):568-78.

928 82. Fuchshofer R. The pathogenic role of transforming growth factor-beta2 in glaucomatous 929 damage to the optic nerve head. Exp Eye Res. 2011;93(2):165-9.

930 83. Fuchshofer R, Tamm ER. The role of TGF-beta in the pathogenesis of primary open-angle 931 glaucoma. Cell Tissue Res. 2012;347(1):279-90. 
932

933

934

935

936

937

938

939

940

941

942

943

944

945

946

947

948

949

950

951

952

953

954

955

956

957

958

959

960

961

962

963

964

965

966

967

968

969

970

971

972

84. Zode GS, Sethi A, Brun-Zinkernagel AM, Chang IF, Clark AF, Wordinger RJ. Transforming growth factor-beta2 increases extracellular matrix proteins in optic nerve head cells via activation of the Smad signaling pathway. Mol Vis. 2011;17:1745-58.

85. Mohammad G, Kowluru RA. Matrix metalloproteinase-2 in the development of diabetic retinopathy and mitochondrial dysfunction. Lab Invest. 2010;90(9):1365-72.

86. Tan W, Zou JL, Yoshida S, Jiang B, Zhou YD. Increased vitreal levels of interleukin-10 in diabetic retinopathy: a Meta-analysis. Int J Ophthalmol. 2020;13(9):1477-83.

87. Dace DS, Khan AA, Kelly J, Apte RS. Interleukin-10 promotes pathological angiogenesis by regulating macrophage response to hypoxia during development. PLoS One. 2008;3(10):e3381.

88. Luzina IG, Keegan AD, Heller NM, Rook GA, Shea-Donohue T, Atamas SP. Regulation of inflammation by interleukin-4: a review of "alternatives". J Leukoc Biol. 2012;92(4):753-64.

89. Major J, Fletcher JE, Hamilton TA. IL-4 pretreatment selectively enhances cytokine and chemokine production in lipopolysaccharide-stimulated mouse peritoneal macrophages. J Immunol. 2002;168(5):2456-63.

90. Gadani SP, Cronk JC, Norris GT, Kipnis J. IL-4 in the brain: a cytokine to remember. J Immunol. 2012;189(9):4213-9.

91. Yoshida S, Sotozono C, Ikeda T, Kinoshita S. Interleukin-6 (IL-6) production by cytokinestimulated human Muller cells. Curr Eye Res. 2001;22(5):341-7.

92. Yao Y, Li R, Du J, Long L, Li X, Luo N. Interleukin-6 and Diabetic Retinopathy: A Systematic Review and Meta-Analysis. Curr Eye Res. 2019;44(5):564-74.

93. Liu X, Ye F, Xiong H, Hu DN, Limb GA, Xie T, et al. IL-1beta induces IL-6 production in retinal Muller cells predominantly through the activation of $\mathrm{p} 38$ MAPK/NF-kappaB signaling pathway. Exp Cell Res. 2015;331(1):223-31.

94. Coughlin BA, Trombley BT, Mohr S. Interleukin-6 (IL-6) mediates protection against glucose toxicity in human Muller cells via activation of VEGF-A signaling. Biochem Biophys Res Commun. 2019;517(2):227-32.

95. Ucgun NI, Zeki-Fikret C, Yildirim Z. Inflammation and diabetic retinopathy. Mol Vis. 2020;26:718-21.

96. Wu H, Hwang DK, Song X, Tao Y. Association between Aqueous Cytokines and Diabetic Retinopathy Stage. J Ophthalmol. 2017;2017:9402198.

97. Bazan JF, Bacon KB, Hardiman G, Wang W, Soo K, Rossi D, et al. A new class of membrane-bound chemokine with a CX3C motif. Nature. 1997;385(6617):640-4.

98. Imai T, Hieshima K, Haskell C, Baba M, Nagira M, Nishimura M, et al. Identification and molecular characterization of fractalkine receptor CX3CR1, which mediates both leukocyte migration and adhesion. Cell. 1997;91(4):521-30.

99. Abu El-Asrar AM, Nawaz MI, Ahmad A, De Zutter A, Siddiquei MM, Blanter M, et al. Evaluation of Proteoforms of the Transmembrane Chemokines CXCL16 and CX3CL1, Their Receptors, and Their Processing Metalloproteinases ADAM10 and ADAM17 in Proliferative Diabetic Retinopathy. Front Immunol. 2020;11:601639.

100. Serra AM, Waddell J, Manivannan A, Xu H, Cotter M, Forrester JV. CD11b+ bone marrowderived monocytes are the major leukocyte subset responsible for retinal capillary leukostasis in

This is a provisional file, not the final typeset article 
experimental diabetes in mouse and express high levels of CCR5 in the circulation. Am J Pathol. 2012;181(2):719-27.

975 101. Wu F, Phone A, Lamy R, Ma D, Laotaweerungsawat S, Chen Y, et al. Correlation of Aqueous, Vitreous, and Plasma Cytokine Levels in Patients With Proliferative Diabetic Retinopathy. Invest Ophthalmol Vis Sci. 2020;61(2):26.

981 103. Greene JA, Portillo JA, Lopez Corcino Y, Subauste CS. CD40-TRAF Signaling Upregulates 982 CX3CL1 and TNF-alpha in Human Aortic Endothelial Cells but Not in Retinal Endothelial Cells. 983 PLoS One. 2015;10(12):e0144133.

984 104. Pauly D, Agarwal D, Dana N, Schafer N, Biber J, Wunderlich KA, et al. Cell-Type-Specific 985 Complement Expression in the Healthy and Diseased Retina. Cell Rep. 2019;29(9):2835-48 e4.

986 105. Kaczmarek-Hajek K, Zhang J, Kopp R, Grosche A, Rissiek B, Saul A, et al. Re-evaluation of neuronal P2X7 expression using novel mouse models and a P2X7-specific nanobody. Elife. 2018;7. e34. histocompatibility antigens by human retinal glial cells. Exp Eye Res. 1991;53(5):603-7. 108. Kambayashi T, Laufer TM. Atypical MHC class II-expressing antigen-presenting cells: can anything replace a dendritic cell? Nat Rev Immunol. 2014;14(11):719-30. Morcillo J. Muller glia and phagocytosis of cell debris in retinal tissue. J Anat. 2017;231(4):471-83. mouse model of retinal degenerative disease. FASEB J. 2019;33(3):3680-92. antimicrobial peptides, oxidative burst and phagocytosis. J Neuroinflammation. 2014;11:33.

1001 112. Gutierrez-Martinez E, Planes R, Anselmi G, Reynolds M, Menezes S, Adiko AC, et al. Cross1002 Presentation of Cell-Associated Antigens by MHC Class I in Dendritic Cell Subsets. Front Immunol. $10032015 ; 6: 363$.

1004 113. Larsson M, Fonteneau JF, Bhardwaj N. Dendritic cells resurrect antigens from dead cells. 1005 Trends Immunol. 2001;22(3):141-8.

1006 114. Kastelan S, Tomic M, Salopek-Rabatic J, Pavan J, Lukenda A, Gotovac M, et al. The association between the HLA system and retinopathy development in patients with type 1 diabetes mellitus. Coll Antropol. 2013;37 Suppl 1:65-70. 53.

1012 116. Dornan TL, Ting A, McPherson CK, Peckar CO, Mann JI, Turner RC, et al. Genetic 1013 susceptibility to the development of retinopathy in insulin-dependent diabetics. Diabetes.

1014 1982;31(3):226-31. 
1015

1016

1017

1018

1019

1020

1021

1022

1023

1024

1025

1026

1027

1028

1029

1030

1031

1032

1033

1034

1035

1036

1037

1038

1039

1040

1041

1042

1043

1044

1045

1046

1047

1048

1049

1050

1051

1052

1053

1054

1055

117. Falck AA, Knip JM, Ilonen JS, Laatikainen LT. Genetic markers in early diabetic retinopathy of adolescents with type I diabetes. J Diabetes Complications. 1997;11(4):203-7.

118. Cruickshanks KJ, Vadheim CM, Moss SE, Roth MP, Riley WJ, Maclaren NK, et al. Genetic marker associations with proliferative retinopathy in persons diagnosed with diabetes before $30 \mathrm{yr}$ of age. Diabetes. 1992;41(7):879-85.

119. Birinci A, Birinci H, Abidinoglu R, Durupinar B, Oge I. Diabetic retinopathy and HLA antigens in type 2 diabetes mellitus. Eur J Ophthalmol. 2002;12(2):89-93.

120. Agardh D, Gaur LK, Agardh E, Landin-Olsson M, Agardh CD, Lernmark A. HLADQB $1 * 0201 / 0302$ is associated with severe retinopathy in patients with IDDM. Diabetologia. 1996;39(11):1313-7.

121. Santos DC, Porto LC, Pizarro MH, de Melo LGN, Silva DA, Oliveira RV, et al. Human Leukocyte Antigens class II (HLA II) gene profile from an admixed population of patients with type 1 diabetes with severe diabetic retinopathy: a nested case-control study in Brazil. Diabetol Metab Syndr. 2021;13(1):83.

122. Liu C, Chen G, Bentley AR, Doumatey A, Zhou J, Adeyemo A, et al. Genome-wide association study for proliferative diabetic retinopathy in Africans. NPJ Genom Med. 2019;4:20.

123. Portillo JA, Greene JA, Okenka G, Miao Y, Sheibani N, Kern TS, et al. CD40 promotes the development of early diabetic retinopathy in mice. Diabetologia. 2014;57(10):2222-31.

124. Portillo JC, Lopez Corcino Y, Miao Y, Tang J, Sheibani N, Kern TS, et al. CD40 in Retinal Müller Cells Induces P2X7-Dependent Cytokine Expression in Macrophages/Microglia in Diabetic Mice and Development of Early Experimental Diabetic Retinopathy. Diabetes. 2017;66(2):483-93.

125. Subauste CS. The CD40-ATP-P2X 7 Receptor Pathway: Cell to Cell Cross-Talk to Promote Inflammation and Programmed Cell Death of Endothelial Cells. Front Immunol. 2019;10:2958.

126. Cvitkovic K, Sesar A, Sesar I, Pusic-Sesar A, Pejic R, Kelava T, et al. Concentrations of Selected Cytokines and Vascular Endothelial Growth Factor in Aqueous Humor and Serum of Diabetic Patients. Semin Ophthalmol. 2020;35(2):126-33.

127. Ozay Y, Ozek D, Yildirim F, Yildirim Z. The effect of diabetes on vitreous levels of adiponectin and inflammatory cytokines in experimental rat model. Adv Clin Exp Med. 2020;29(4):449-52.

128. Vujosevic S, Micera A, Bini S, Berton M, Esposito G, Midena E. Proteome analysis of retinal glia cells-related inflammatory cytokines in the aqueous humour of diabetic patients. Acta Ophthalmol. 2016;94(1):56-64.

129. Tan L, Gordon KB, Mueller JP, Matis LA, Miller SD. Presentation of proteolipid protein epitopes and B7-1-dependent activation of encephalitogenic T cells by IFN-gamma-activated SJL/J astrocytes. J Immunol. 1998;160(9):4271-9.

130. Portillo JC, Yu JS, Hansen S, Kern TS, Subauste MC, Subauste CS. A cell-penetrating CD40TRAF2,3 blocking peptide diminishes inflammation and neuronal loss after ischemia/reperfusion. FASEB J. 2021;35(3):e21412.

131. Cecilia OM, Jose Alberto CG, Jose NP, Ernesto German CM, Ana Karen LC, Luis Miguel RP, et al. Oxidative Stress as the Main Target in Diabetic Retinopathy Pathophysiology. J Diabetes Res. 2019;2019:8562408.

This is a provisional file, not the final typeset article 
1056

1057

1058

1059

1060

1061

1062

1063

1064

1065

1066

1067

1068

1069

1070

1071

1072

1073

1074

1075

1076

1077

1078

1079

1080

1081

1082

1083

1084

1085

1086

1087

1088

1089

1090

132. Tien T, Zhang J, Muto T, Kim D, Sarthy VP, Roy S. High Glucose Induces Mitochondrial Dysfunction in Retinal Muller Cells: Implications for Diabetic Retinopathy. Invest Ophthalmol Vis Sci. 2017;58(7):2915-21.

133. Krugel K, Wurm A, Pannicke T, Hollborn M, Karl A, Wiedemann P, et al. Involvement of oxidative stress and mitochondrial dysfunction in the osmotic swelling of retinal glial cells from diabetic rats. Exp Eye Res. 2011;92(1):87-93.

134. Behl T, Kotwani A. Exploring the various aspects of the pathological role of vascular endothelial growth factor (VEGF) in diabetic retinopathy. Pharmacol Res. 2015;99:137-48.

135. Homme RP, Singh M, Majumder A, George AK, Nair K, Sandhu HS, et al. Remodeling of Retinal Architecture in Diabetic Retinopathy: Disruption of Ocular Physiology and Visual Functions by Inflammatory Gene Products and Pyroptosis. Front Physiol. 2018;9:1268.

136. Wang J, Shanmugam A, Markand S, Zorrilla E, Ganapathy V, Smith SB. Sigma 1 receptor regulates the oxidative stress response in primary retinal Muller glial cells via NRF2 signaling and system xc(-), the $\mathrm{Na}(+)$-independent glutamate-cystine exchanger. Free Radic Biol Med. 2015;86:2536.

137. Geng J, Sun X, Wang P, Zhang S, Wang X, Wu H, et al. Kinases Mst1 and Mst2 positively regulate phagocytic induction of reactive oxygen species and bactericidal activity. Nat Immunol. 2015;16(11):1142-52.

138. Wang P, Geng J, Gao J, Zhao H, Li J, Shi Y, et al. Macrophage achieves self-protection against oxidative stress-induced ageing through the Mst-Nrf2 axis. Nat Commun. 2019;10(1):755.

139. Hollborn M, Ulbricht E, Rillich K, Dukic-Stefanovic S, Wurm A, Wagner L, et al. The human Muller cell line MIO-M1 expresses opsins. Mol Vis. 2011;17:2738-50.

140. Lawrence JM, Singhal S, Bhatia B, Keegan DJ, Reh TA, Luthert PJ, et al. MIO-M1 cells and similar muller glial cell lines derived from adult human retina exhibit neural stem cell characteristics. Stem Cells. 2007;25(8):2033-43.

141. Merl J, Ueffing M, Hauck SM, von Toerne C. Direct comparison of MS-based label-free and SILAC quantitative proteome profiling strategies in primary retinal Muller cells. Proteomics. 2012;12(12):1902-11.

142. Hauck SM, Gloeckner CJ, Harley ME, Schoeffmann S, Boldt K, Ekstrom PA, et al. Identification of paracrine neuroprotective candidate proteins by a functional assay-driven proteomics approach. Mol Cell Proteomics. 2008;7(7):1349-61.

143. von Toerne C, Menzler J, Ly A, Senninger N, Ueffing M, Hauck SM. Identification of a novel neurotrophic factor from primary retinal Muller cells using stable isotope labeling by amino acids in cell culture (SILAC). Mol Cell Proteomics. 2014;13(9):2371-81.

This is a provisional file, not the final typeset article 